\title{
Finite Theories Before and After the Discovery of a Higgs Boson at the LHC
}

\author{
S. Heinemeyer 谋, M. Mondragón讯 And G. Zoupanos讯 \\ ${ }^{1}$ Instituto de Física de Cantabria (CSIC-UC), Santander, Spain \\ ${ }^{2}$ Instituto de Física, Universidad Nacional Autónoma de México \\ Apdo. Postal 20-364, México 01000 D.F., México \\ ${ }^{3}$ Arnold-Sommerfeld-Center für Theoretische Physik, Department für Physik, \\ Ludwig-Maximilians-Universität München, Theresienstrasse 37, 80333 München, Germany \\ Max-Planck-Institut für Physik (Werner-Heisenberg-Institut) Föhringer Ring 6, \\ 80805 München, Germany \\ Departamento de Física Teórica and Instituto de Física Teórica, IFT-UAM/CSIC \\ Universidad Autónoma de Madrid, Cantoblanco, Madrid, Spain
}

\begin{abstract}
Finite Unified Theories (FUTs) are $N=1$ supersymmetric Grand Unified Theories (GUTs) which can be made finite to all-loop orders, based on the principle of reduction of couplings, and therefore are provided with a large predictive power. Confronting the predictions of SU(5) FUTs with the top and bottom quark masses and other lowenergy experimental constraints a light Higgs-boson mass in the range $M_{h} \sim 121-126$ $\mathrm{GeV}$ was predicted, in striking agreement with the recent discovery of a Higgs-like state around $\sim 125.5 \mathrm{GeV}$ at ATLAS and CMS. Furthermore the favoured model, a finiteness constrained version of the MSSM, naturally predicts a relatively heavy spectrum with coloured supersymmetric particles above $\sim 1.5 \mathrm{TeV}$, consistent with the non-observation of those particles at the LHC. Restricting further the best FUT's parameter space according to the discovery of a Higgs-like state and B-physics observables we find predictions for the rest of the Higgs masses and the supersymmetric particle spectrum.
\end{abstract}

*email: Sven.Heinemeyer@cern.ch

†email: myriam@fisica.unam.mx

$\ddagger$ email: George.Zoupanos@cern.ch

$\S$ On leave from Physics Department, National Technical University, Zografou Campus:

Heroon Polytechniou 9, 15780 Zografou, Athens, Greece 


\section{The idea}

A large and sustained effort has been done in the recent years aiming to achieve a unified description of all interactions. Out of this endeavor two main directions have emerged as the most promising to attack the problem, namely, the superstring theories and non-commutative geometry. The two approaches, although at a different stage of development, have common unification targets and share similar hopes for exhibiting improved renormalization properties in the ultraviolet (UV) as compared to ordinary field theories. Moreover the two frameworks came closer by the observation that a natural realization of non-commutativity of space appears in the string theory context of D-branes in the presence of a constant background antisymmetric field [1]. Among the numerous important developments in both frameworks, it is worth noting two conjectures of utmost importance that signal the developments in certain directions in string theory, although not exclusively there, related related to the main theme of the present review. The conjectures refer to (i) the duality among the 4dimensional $N=4$ supersymmetric Yang-Mills theory and the type IIB string theory on $A d S_{5} \times S^{5}$ [2]; the former being the maximal $N=4$ supersymmetric Yang-Mills theory is known to be UV all-loop finite theory [3,4], (ii) the possibility of "miraculous" UV divergence cancellations in 4-dimensional maximal $N=8$ supergravity leading to a finite theory, as has been confirmed in a remarkable 4-loop calculation [5] 9]. However, despite the importance of having frameworks to discuss quantum gravity in a self-consistent way and possibly to construct finite theories in these type of frameworks, it is also very interesting to search for the minimal realistic framework in which finiteness can take place. After all the history of our field teaches us that if a new idea works, it does that in its simplest form. In addition, the main goal expected from a unified description of interactions by the particle physics community is to understand the present day large number of free parameters of the Standard Model (SM) in terms of a few fundamental ones. In other words, to achieve reduction of couplings at a more fundamental level.

To reduce the number of free parameters of a theory, and thus render it more predictive, one is usually led to introduce a symmetry. Grand Unified Theories (GUTs) are very good examples of such a procedure [10 14. For instance, in the case of minimal $S U(5)$, because of (approximate) gauge coupling unification, it was possible to reduce the gauge couplings by one and give a prediction for one of them. In fact, LEP data [15] seem to suggest that a further symmetry, namely $N=1$ global supersymmetry (SUSY) [16, 17] should also be required to make the prediction viable. GUTs can also relate the Yukawa couplings among themselves, again $S U(5)$ provided an example of this by predicting the ratio $M_{\tau} / M_{b}$ [18] in the SM. Unfortunately, requiring more gauge symmetry does not seem to help, since additional complications are introduced due to new degrees of freedom, in the ways and channels of breaking the symmetry, and so on.

A natural extension of the GUT idea is to find a way to relate the gauge and Yukawa sectors of a theory, that is to achieve Gauge-Yukawa Unification (GYU) [19 21]. A symmetry which naturally relates the two sectors is supersymmetry, in particular $N=2$ SUSY [22]. It turns out, however, that $N=2$ supersymmetric theories have serious phenomenological problems due to light mirror fermions. Also in superstring theories and in composite models there exist relations among the gauge and Yukawa couplings, but both kind of theories have phenomenological problems, which we are not going to address here. 
In our studies [19 21,23 28] we have developed a complementary strategy in searching for a more fundamental theory possibly at the Planck scale, whose basic ingredients are GUTs and supersymmetry, but its consequences certainly go beyond the known ones. Our method consists of hunting for renormalization group invariant (RGI) relations holding below the Planck scale, which in turn are preserved down to the GUT scale. This programme, called Gauge-Yukawa unification scheme, applied in the dimensionless couplings of supersymmetric GUTs, such as gauge and Yukawa couplings, had already noticable successes by predicting correctly, among others, the top quark mass in the finite and in the minimal $N=1$ supersymmetric SU(5) GUTs [23 25]. An impressive aspect of the RGI relations is that one can guarantee their validity to all-orders in perturbation theory by studying the uniqueness of the resulting relations at one-loop, as was proven [29, 30] in the early days of the programme of reduction of couplings [29 34. Even more remarkable is the fact that it is possible to find RGI relations among couplings that guarantee finiteness to all-orders in perturbation theory 3539 .

It is worth noting that the above principles have only been applied in supersymmetric GUTs for reasons that will be transparent in the following sections. We should also stress that our conjecture for GYU is by no means in conflict with earlier interesting proposals, but it rather uses all of them, hopefully in a more successful perspective. For instance, the use of SUSY GUTs comprises the demand of the cancellation of quadratic divergences in the SM. Similarly, the very interesting conjectures about the infrared fixed points are generalized in our proposal, since searching for RGI relations among various couplings corresponds to searching for fixed points of the coupled differential equations obeyed by the various couplings of a theory.

Although SUSY seems to be an essential feature for a successful realization of the above programme, its breaking has to be understood too, since it has the ambition to supply the SM with predictions for several of its free parameters. Indeed, the search for RGI relations has been extended to the soft SUSY breaking sector (SSB) of these theories [28, 40], which involves parameters of dimension one and two. A breakthrough concerning the renormalization properties of the SSB was made [41 47], based concepturally and technically on the work of Ref. [48]: the powerful supergraph method [49 52] for studying supersymmetric theories was applied to the softly broken ones by using the "spurion" external space-time independent superfields [53]. In the latter method a softly broken supersymmetric gauge theory is considered as a supersymmetric one in which the various parameters such as couplings and masses have been promoted to external superfields that acquire "vacuum expectation values". Based on this method the relations among the soft term renormalization and that of an unbroken supersymmetric theory were derived. In particular the $\beta$-functions of the parameters of the softly broken theory are expressed in terms of partial differential operators involving the dimensionless parameters of the unbroken theory. The key point in the strategy of Refs. [44 47] in solving the set of coupled differential equations so as to be able to express all parameters in a RGI way, was to transform the partial differential operators involved to total derivative operators. This is indeed possible to be done on the RGI surface which is defined by the solution of the reduction equations.

On the phenomenological side there exist some serious developments, too. Previously an appealing "universal" set of soft scalar masses was asummed in the SSB sector of supersymmetric theories, given that apart from economy and simplicity (1) they are part of 
the constraints that preserve finiteness up to two-loops [54, 55], (2) they are RGI up to two-loops in more general supersymmetric gauge theories, subject to the condition known as $P=1 / 3 Q$ [40], and (3) they appear in the attractive dilaton dominated SUSY breaking superstring scenarios [56 58. However, further studies have exhibited a number of problems all due to the restrictive nature of the "universality" assumption for the soft scalar masses. For instance, (a) in finite unified theories the universality predicts that the lightest supersymmetric particle is a charged particle, namely the superpartner of the $\tau$ lepton $\tilde{\tau}$, (b) the Minimal Supersymmetric Standard Model (MSSM) with universal soft scalar masses is inconsistent with the attractive radiative electroweak symmetry breaking [58], and (c) which is the worst of all, the universal soft scalar masses lead to charge and/or colour breaking minima deeper than the standard vacuum [59]. Therefore, there have been attempts to relax this constraint without loosing its attractive features. First, an interesting observation was made that in $N=1$ Gauge-Yukawa unified theories there exists a RGI sum rule for the soft scalar masses at lower orders; at one-loop for the non-finite case [60] and at two-loops for the finite case [61]. The sum rule manages to overcome the above unpleasant phenomenological consequences. Moreover it was proven [47] that the sum rule for the soft scalar massses is RGI to all-orders for both the general as well as for the finite case. Finally, the exact $\beta$-function for the soft scalar masses in the Novikov-Shifman-Vainstein-Zakharov (NSVZ) scheme [62 64] for the softly broken supersymmetric QCD has been obtained [47.

Armed with the above tools and results we are in a position to study and predict the spectrum of the full finite models in terms of few input parameters. In particular, a prediction for the lightest MSSM Higgs boson can be obtained. It turned out that the prediction is naturally in very good agreement with the discovery of a Higgs-like particle at the LHC [65, 66] at around $\sim 126 \mathrm{GeV}$. Identifying the lightest Higgs boson with the newly discovered state one can restrict the allowed parameter space of the model. We review how this reduction of parameter space impacts the prediction of the SUSY spectrum and the discovery potential of the LHC and future $e^{+} e^{-}$colliders.

\section{Theoretical basis}

In this section we outline the idea of reduction of couplings. Any RGI relation among couplings (which does not depend on the renormalization scale $\mu$ explicitly) can be expressed, in the implicit form $\Phi\left(g_{1}, \cdots, g_{A}\right)=$ const., which has to satisfy the partial differential equation (PDE)

$$
\mu \frac{d \Phi}{d \mu}=\vec{\nabla} \cdot \vec{\beta}=\sum_{a=1}^{A} \beta_{a} \frac{\partial \Phi}{\partial g_{a}}=0
$$

where $\beta_{a}$ is the $\beta$-function of $g_{a}$. This PDE is equivalent to a set of ordinary differential equations, the so-called reduction equations (REs) [29, 30,67],

$$
\beta_{g} \frac{d g_{a}}{d g}=\beta_{a}, a=1, \cdots, A
$$

where $g$ and $\beta_{g}$ are the primary coupling and its $\beta$-function, and the counting on $a$ does not include $g$. Since maximally $(A-1)$ independent RGI "constraints" in the $A$-dimensional 
space of couplings can be imposed by the $\Phi_{a}$ 's, one could in principle express all the couplings in terms of a single coupling $g$. The strongest requirement is to demand power series solutions to the REs,

$$
g_{a}=\sum_{n} \rho_{a}^{(n)} g^{2 n+1}
$$

which formally preserve perturbative renormalizability. Remarkably, the uniqueness of such power series solutions can be decided already at the one-loop level [29, 30, 67]. To illustrate this, let us assume that the $\beta$-functions have the form

$$
\begin{aligned}
& \beta_{a}=\frac{1}{16 \pi^{2}}\left[\sum_{b, c, d \neq g} \beta_{a}^{(1) b c d} g_{b} g_{c} g_{d}+\sum_{b \neq g} \beta_{a}^{(1) b} g_{b} g^{2}\right]+\cdots \\
& \beta_{g}=\frac{1}{16 \pi^{2}} \beta_{g}^{(1)} g^{3}+\cdots
\end{aligned}
$$

where $\cdots$ stands for higher order terms, and $\beta_{a}^{(1) b c d}$, s are symmetric in $b, c, d$. We then assume that the $\rho_{a}^{(n)}$ 's with $n \leq r$ have been uniquely determined. To obtain $\rho_{a}^{(r+1)}$, $\mathrm{s}$, we insert the power series (3) into the REs (2) and collect terms of $\mathcal{O}\left(g^{2 r+3}\right)$ and find

$$
\sum_{d \neq g} M(r)_{a}^{d} \rho_{d}^{(r+1)}=\text { lower order quantities }
$$

where the r.h.s. is known by assumption, and

$$
\begin{aligned}
M(r)_{a}^{d} & =3 \sum_{b, c \neq g} \beta_{a}^{(1) b c d} \rho_{b}^{(1)} \rho_{c}^{(1)}+\beta_{a}^{(1) d}-(2 r+1) \beta_{g}^{(1)} \delta_{a}^{d}, \\
0 & =\sum_{b, c, d \neq g} \beta_{a}^{(1) b c d} \rho_{b}^{(1)} \rho_{c}^{(1)} \rho_{d}^{(1)}+\sum_{d \neq g} \beta_{a}^{(1) d} \rho_{d}^{(1)}-\beta_{g}^{(1)} \rho_{a}^{(1)},
\end{aligned}
$$

Therefore, the $\rho_{a}^{(n)}$ 's for all $n>1$ for a given set of $\rho_{a}^{(1)}$ 's can be uniquely determined if $\operatorname{det} M(n)_{a}^{d} \neq 0$ for all $n \geq 0$.

As it will be clear later by examining specific examples, the various couplings in supersymmetric theories have the same asymptotic behaviour. Therefore searching for a power series solution of the form (3) to the REs (2) is justified. This is not the case in non-supersymmetric theories, although the deeper reason for this fact is not fully understood.

The possibility of coupling unification described in this section is without any doubt attractive because the "completely reduced" theory contains only one independent coupling, but it can be unrealistic. Therefore, one often would like to impose fewer RGI constraints, and this is the idea of partial reduction [68, 69].

\subsection{Reduction of dimensionful parameters}

The reduction of couplings was originally formulated for massless theories on the basis of the Callan-Symanzik equation [29, 30,67]. The extension to theories with massive parameters is not straightforward if one wants to keep the generality and the rigor on the same level as for 
the massless case; one has to fulfill a set of requirements coming from the renormalization group equations, the Callan-Symanzik equations, etc. along with the normalization conditions imposed on irreducible Green's functions [70]. See [71] for interesting results in this direction. Here to simplify the situation and following Ref. [28] we would like to assume that a mass-independent renormalization scheme has been employed so that all the RG functions have only trivial dependencies of dimensional parameters.

To be general, we consider a renormalizable theory which contains a set of $(N+1)$ dimension-zero couplings, $\left\{\hat{g}_{0}, \hat{g}_{1}, \ldots, \hat{g}_{N}\right\}$, a set of $L$ parameters with dimension one, $\left\{\hat{h}_{1}, \ldots, \hat{h}_{L}\right\}$, and a set of $M$ parameters with dimension two, $\left\{\hat{m}_{1}^{2}, \ldots, \hat{m}_{M}^{2}\right\}$. The renormalized irreducible vertex function satisfies the RG equation

$$
\begin{aligned}
0 & =\mathcal{D} \Gamma\left[\boldsymbol{\Phi}^{\prime} s ; \hat{g}_{0}, \hat{g}_{1}, \ldots, \hat{g}_{N} ; \hat{h}_{1}, \ldots, \hat{h}_{L} ; \hat{m}_{1}^{2}, \ldots, \hat{m}_{M}^{2} ; \mu\right] \\
\mathcal{D} & =\mu \frac{\partial}{\partial \mu}+\sum_{i=0}^{N} \beta_{i} \frac{\partial}{\partial \hat{g}_{i}}+\sum_{a=1}^{L} \gamma_{a}^{h} \frac{\partial}{\partial \hat{h}_{a}}+\sum_{\alpha=1}^{M} \gamma_{\alpha}^{m^{2}} \frac{\partial}{\partial \hat{m}_{\alpha}^{2}}+\sum_{J} \Phi_{I} \gamma^{\phi I} \frac{\delta}{\delta \Phi_{J}} .
\end{aligned}
$$

Since we assume a mass-independent renormalization scheme, the $\gamma$ 's have the form

$$
\begin{aligned}
\gamma_{a}^{h} & =\sum_{b=1}^{L} \gamma_{a}^{h, b}\left(g_{0}, \ldots, g_{N}\right) \hat{h}_{b}, \\
\gamma_{\alpha}^{m^{2}} & =\sum_{\beta=1}^{M} \gamma_{\alpha}^{m^{2}, \beta}\left(g_{0}, \ldots, g_{N}\right) \hat{m}_{\beta}^{2}+\sum_{a, b=1}^{L} \gamma_{\alpha}^{m^{2}, a b}\left(g_{0}, \ldots, g_{N}\right) \hat{h}_{a} \hat{h}_{b},
\end{aligned}
$$

where $\gamma_{a}^{h, b}, \gamma_{\alpha}^{m^{2}, \beta}$ and $\gamma_{a}^{m^{2}, a b}$ are power series of the dimension-zero couplings $g$ 's in perturbation theory.

As in the massless case, we then look for conditions under which the reduction of parameters,

$$
\begin{aligned}
\hat{g}_{i} & =\hat{g}_{i}(g),(i=1, \ldots, N), \\
\hat{h}_{a} & =\sum_{b=1}^{P} f_{a}^{b}(g) h_{b},(a=P+1, \ldots, L), \\
\hat{m}_{\alpha}^{2} & =\sum_{\beta=1}^{Q} e_{\alpha}^{\beta}(g) m_{\beta}^{2}+\sum_{a, b=1}^{P} k_{\alpha}^{a b}(g) h_{a} h_{b},(\alpha=Q+1, \ldots, M),
\end{aligned}
$$

is consistent with the RG equation (1), where we assume that $g \equiv g_{0}, h_{a} \equiv \hat{h}_{a} \quad(1 \leq a \leq P)$ and $m_{\alpha}^{2} \equiv \hat{m}_{\alpha}^{2} \quad(1 \leq \alpha \leq Q)$ are independent parameters of the reduced theory. We find that the following set of equations has to be satisfied:

$$
\begin{aligned}
\beta_{g} \frac{\partial \hat{g}_{i}}{\partial g} & =\beta_{i},(i=1, \ldots, N), \\
\beta_{g} \frac{\partial \hat{h}_{a}}{\partial g}+\sum_{b=1}^{P} \gamma_{b}^{h} \frac{\partial \hat{h}_{a}}{\partial h_{b}} & =\gamma_{a}^{h},(a=P+1, \ldots, L),
\end{aligned}
$$




$$
\beta_{g} \frac{\partial \hat{m}_{\alpha}^{2}}{\partial g}+\sum_{a=1}^{P} \gamma_{a}^{h} \frac{\partial \hat{m}_{\alpha}^{2}}{\partial h_{a}}+\sum_{\beta=1}^{Q} \gamma_{\beta}^{m^{2}} \frac{\partial \hat{m}_{\alpha}^{2}}{\partial m_{\beta}^{2}}=\gamma_{\alpha}^{m^{2}},(\alpha=Q+1, \ldots, M)
$$

Using eq.(17) for $\gamma$ 's, one finds that eqs.(12,-14) reduce to

$$
\begin{aligned}
& \beta_{g} \frac{d f_{a}^{b}}{d g}+\sum_{c=1}^{P} f_{a}^{c}\left[\gamma_{c}^{h, b}+\sum_{d=P+1}^{L} \gamma_{c}^{h, d} f_{d}^{b}\right]-\gamma_{a}^{h, b}-\sum_{d=P+1}^{L} \gamma_{a}^{h, d} f_{d}^{b}=0 \\
& (a=P+1, \ldots, L ; b=1, \ldots, P) \\
& \beta_{g} \frac{d e_{\alpha}^{\beta}}{d g}+\sum_{\gamma=1}^{Q} e_{\alpha}^{\gamma}\left[\gamma_{\gamma}^{m^{2}, \beta}+\sum_{\delta=Q+1}^{M} \gamma_{\gamma}^{m^{2}, \delta} e_{\delta}^{\beta}\right]-\gamma_{\alpha}^{m^{2}, \beta}-\sum_{\delta=Q+1}^{M} \gamma_{\alpha}^{m^{2}, \delta} e_{\delta}^{\beta}=0 \\
& (\alpha=Q+1, \ldots, M ; \beta=1, \ldots, Q), \\
& \beta_{g} \frac{d k_{\alpha}^{a b}}{d g}+2 \sum_{c=1}^{P}\left(\gamma_{c}^{h, a}+\sum_{d=P+1}^{L} \gamma_{c}^{h, d} f_{d}^{a}\right) k_{\alpha}^{c b}+\sum_{\beta=1}^{Q} e_{\alpha}^{\beta}\left[\gamma_{\beta}^{m^{2}, a b}+\sum_{c, d=P+1}^{L} \gamma_{\beta}^{m^{2}, c d} f_{c}^{a} f_{d}^{b}\right. \\
& \left.+2 \sum_{c=P+1}^{L} \gamma_{\beta}^{m^{2}, c b} f_{c}^{a}+\sum_{\delta=Q+1}^{M} \gamma_{\beta}^{m^{2}, \delta} k_{\delta}^{a b}\right]-\left[\gamma_{\alpha}^{m^{2}, a b}+\sum_{c, d=P+1}^{L} \gamma_{\alpha}^{m^{2}, c d} f_{c}^{a} f_{d}^{b}\right. \\
& \left.+2 \sum_{c=P+1}^{L} \gamma_{\alpha}^{m^{2}, c b} f_{c}^{a}+\sum_{\delta=Q+1}^{M} \gamma_{\alpha}^{m^{2}, \delta} k_{\delta}^{a b}\right]=0, \\
& (\alpha=Q+1, \ldots, M ; a, b=1, \ldots, P) .
\end{aligned}
$$

If these equations are satisfied, the irreducible vertex function of the reduced theory

$$
\begin{aligned}
& \Gamma_{R}\left[\boldsymbol{\Phi}^{\prime} s ; g ; h_{1}, \ldots, h_{P} ; m_{1}^{2}, \ldots, \hat{m}_{Q}^{2} ; \mu\right] \\
\equiv & \Gamma\left[\boldsymbol{\Phi}^{\prime} s ; g, \hat{g}_{1}(g), \ldots, \hat{g}_{N}(g) ; h_{1}, \ldots, h_{P}, \hat{h}_{P+1}(g, h), \ldots, \hat{h}_{L}(g, h) ;\right. \\
& \left.m_{1}^{2}, \ldots, \hat{m}_{Q}^{2}, \hat{m}_{Q+1}^{2}\left(g, h, m^{2}\right), \ldots, \hat{m}_{M}^{2}\left(g, h, m^{2}\right) ; \mu\right]
\end{aligned}
$$

has the same renormalization group flow as the original one.

The requirement for the reduced theory to be perturbative renormalizable means that the functions $\hat{g}_{i}, f_{a}^{b}, e_{\alpha}^{\beta}$ and $k_{\alpha}^{a b}$, defined in eqs. (9-11), should have a power series expansion in the primary coupling $g$ :

$$
\begin{aligned}
& \hat{g}_{i}=g \sum_{n=0}^{\infty} \rho_{i}^{(n)} g^{n}, f_{a}^{b}=g \sum_{n=0}^{\infty} \eta_{a}^{b(n)} g^{n}, \\
& e_{\alpha}^{\beta}=\sum_{n=0}^{\infty} \xi_{\alpha}^{\beta(n)} g^{n}, k_{\alpha}^{a b}=\sum_{n=0}^{\infty} \chi_{\alpha}^{a b(n)} g^{n} .
\end{aligned}
$$

To obtain the expansion coefficients, we insert the power series ansatz above into eqs. [12]15] 17) and require that the equations are satisfied at each order in $g$. Note that the existence of a unique power series solution is a non-trivial matter: it depends on the theory as well as on the choice of the set of independent parameters. 


\section{$2.2 \quad$ Finiteness in $\mathrm{N}=1$ Supersymmetric Gauge Theories}

Let us consider a chiral, anomaly free, $N=1$ globally supersymmetric gauge theory based on a group $\mathrm{G}$ with gauge coupling constant $g$. The superpotential of the theory is given by

$$
W=\frac{1}{2} m_{i j} \phi_{i} \phi_{j}+\frac{1}{6} C_{i j k} \phi_{i} \phi_{j} \phi_{k},
$$

where $m_{i j}$ and $C_{i j k}$ are gauge invariant tensors and the matter field $\phi_{i}$ transforms according to the irreducible representation $R_{i}$ of the gauge group $G$. The renormalization constants associated with the superpotential (20), assuming that SUSY is preserved, are

$$
\begin{aligned}
\phi_{i}^{0} & =\left(Z_{i}^{j}\right)^{(1 / 2)} \phi_{j}, \\
m_{i j}^{0} & =Z_{i j}^{i^{\prime} j^{\prime}} m_{i^{\prime} j^{\prime}}, \\
C_{i j k}^{0} & =Z_{i j k}^{i^{\prime} j^{\prime} k^{\prime}} C_{i^{\prime} j^{\prime} k^{\prime}} .
\end{aligned}
$$

The $N=1$ non-renormalization theorem [51,72, 73] ensures that there are no mass and cubic-interaction-term infinities and therefore

$$
\begin{aligned}
Z_{i j k}^{i^{\prime} j^{\prime} k^{\prime}} Z_{i^{\prime}}^{1 / 2 i^{\prime \prime}} Z_{j^{\prime}}^{1 / 2 j^{\prime \prime}} Z_{k^{\prime}}^{1 / 2 k^{\prime \prime}} & =\delta_{(i}^{i^{\prime \prime}} \delta_{j}^{j^{\prime \prime}} \delta_{k)}^{k^{\prime \prime}}, \\
Z_{i j}^{i^{\prime} j^{\prime}} Z_{i^{\prime}}^{1 / 2 i^{\prime \prime}} Z_{j^{\prime}}^{1 / 2 j^{\prime \prime}} & =\delta_{(i}^{i^{\prime \prime}} \delta_{j}^{j^{\prime \prime}} .
\end{aligned}
$$

As a result the only surviving possible infinities are the wave-function renormalization constants $Z_{i}^{j}$, i.e., one infinity for each field. The one -loop $\beta$-function of the gauge coupling $g$ is given by [74]

$$
\beta_{g}^{(1)}=\frac{d g}{d t}=\frac{g^{3}}{16 \pi^{2}}\left[\sum_{i} l\left(R_{i}\right)-3 C_{2}(G)\right],
$$

where $l\left(R_{i}\right)$ is the Dynkin index of $R_{i}$ and $C_{2}(G)$ is the quadratic Casimir of the adjoint representation of the gauge group $G$. The $\beta$-functions of $C_{i j k}$, by virtue of the non-renormalization theorem, are related to the anomalous dimension matrix $\gamma_{i j}$ of the matter fields $\phi_{i}$ as:

$$
\beta_{i j k}=\frac{d C_{i j k}}{d t}=C_{i j l} \gamma_{k}^{l}+C_{i k l} \gamma_{j}^{l}+C_{j k l} \gamma_{i}^{l} .
$$

At one-loop level $\gamma_{i j}$ is [74]

$$
\gamma_{j}^{i(1)}=\frac{1}{32 \pi^{2}}\left[C^{i k l} C_{j k l}-2 g^{2} C_{2}\left(R_{i}\right) \delta_{j}^{1}\right],
$$

where $C_{2}\left(R_{i}\right)$ is the quadratic Casimir of the representation $R_{i}$, and $C^{i j k}=C_{i j k}^{*}$. Since dimensional coupling parameters such as masses and couplings of cubic scalar field terms do not influence the asymptotic properties of a theory on which we are interested here, it is sufficient to take into account only the dimensionless supersymmetric couplings such as $g$ and $C_{i j k}$. So we neglect the existence of dimensional parameters, and assume furthermore that $C_{i j k}$ are real so that $C_{i j k}^{2}$ always are positive numbers. 
As one can see from Eqs. (25) and (27), all the one-loop $\beta$-functions of the theory vanish if $\beta_{g}^{(1)}$ and $\gamma_{i j}^{(1)}$ vanish, i.e.

$$
\begin{gathered}
\sum_{i} \ell\left(R_{i}\right)=3 C_{2}(G), \\
C^{i k l} C_{j k l}=2 \delta_{j}^{i} g^{2} C_{2}\left(R_{i}\right),
\end{gathered}
$$

The conditions for finiteness for $N=1$ field theories with $S U(N)$ gauge symmetry are discussed in [75], and the analysis of the anomaly-free and no-charge renormalization requirements for these theories can be found in [76]. A very interesting result is that the conditions (28,29) are necessary and sufficient for finiteness at the two-loop level [74, 77, 80].

In case SUSY is broken by soft terms, the requirement of finiteness in the one-loop soft breaking terms imposes further constraints among themselves [54]. In addition, the same set of conditions that are sufficient for one-loop finiteness of the soft breaking terms render the soft sector of the theory two-loop finite [55].

The one- and two-loop finiteness conditions (2829) restrict considerably the possible choices of the irreps. $R_{i}$ for a given group $G$ as well as the Yukawa couplings in the superpotential (20). Note in particular that the finiteness conditions cannot be applied to the minimal supersymmetric standard model (MSSM), since the presence of a $U(1)$ gauge group is incompatible with the condition (28), due to $C_{2}[U(1)]=0$. This naturally leads to the expectation that finiteness should be attained at the grand unified level only, the MSSM being just the corresponding, low-energy, effective theory.

Another important consequence of one- and two-loop finiteness is that SUSY (most probably) can only be broken due to the soft breaking terms. Indeed, due to the unacceptability of gauge singlets, F-type spontaneous symmetry breaking [81] terms are incompatible with finiteness, as well as D-type [82] spontaneous breaking which requires the existence of a $U(1)$ gauge group.

A natural question to ask is what happens at higher loop orders. The answer is contained in a theorem [35,36] which states the necessary and sufficient conditions to achieve finiteness at all orders. Before we discuss the theorem let us make some introductory remarks. The finiteness conditions impose relations between gauge and Yukawa couplings. To require such relations which render the couplings mutually dependent at a given renormalization point is trivial. What is not trivial is to guarantee that relations leading to a reduction of the couplings hold at any renormalization point. As we have seen, the necessary and also sufficient, condition for this to happen is to require that such relations are solutions to the REs

$$
\beta_{g} \frac{d C_{i j k}}{d g}=\beta_{i j k}
$$

and hold at all orders. Remarkably, the existence of all-order power series solutions to (30) can be decided at one-loop level, as already mentioned.

Let us now turn to the all-order finiteness theorem [35, 36], which states that if an $N=1$ supersymmetric gauge theory can become finite to all orders in the sense of vanishing $\beta$ functions, that is of physical scale invariance. It is based on (a) the structure of the supercurrent in $N=1$ supersymmetric gauge theory [83 85], and on (b) the non-renormalization properties of $N=1$ chiral anomalies [35, 36, 86, 88]. Details on the proof can be found in 
refs. [35, 36] and further discussion in Refs. [37, 86 89]. Here, following mostly Ref. [89] we present a comprehensible sketch of the proof.

Consider an $N=1$ supersymmetric gauge theory, with simple Lie group $G$. The content of this theory is given at the classical level by the matter supermultiplets $S_{i}$, which contain a scalar field $\phi_{i}$ and a Weyl spinor $\psi_{i a}$, and the vector supermultiplet $V_{a}$, which contains a gauge vector field $A_{\mu}^{a}$ and a gaugino Weyl spinor $\lambda_{\alpha}^{a}$.

Let us first recall certain facts about the theory:

(1) A massless $N=1$ supersymmetric theory is invariant under a $U(1)$ chiral transformation $R$ under which the various fields transform as follows

$$
\begin{aligned}
A_{\mu}^{\prime} & =A_{\mu}, \quad \lambda_{\alpha}^{\prime}=\exp (-i \theta) \lambda_{\alpha} \\
\phi^{\prime} & =\exp \left(-i \frac{2}{3} \theta\right) \phi, \quad \psi_{\alpha}^{\prime}=\exp \left(-i \frac{1}{3} \theta\right) \psi_{\alpha}, \cdots
\end{aligned}
$$

The corresponding axial Noether current $J_{R}^{\mu}(x)$ is

$$
J_{R}^{\mu}(x)=\bar{\lambda} \gamma^{\mu} \gamma^{5} \lambda+\cdots
$$

is conserved classically, while in the quantum case is violated by the axial anomaly

$$
\partial_{\mu} J_{R}^{\mu}=r\left(\epsilon^{\mu \nu \sigma \rho} F_{\mu \nu} F_{\sigma \rho}+\cdots\right) .
$$

From its known topological origin in ordinary gauge theories [90 92], one would expect the axial vector current $J_{R}^{\mu}$ to satisfy the Adler-Bardeen theorem and receive corrections only at the one-loop level. Indeed it has been shown that the same non-renormalization theorem holds also in supersymmetric theories [86 88]. Therefore

$$
r=\hbar \beta_{g}^{(1)} .
$$

(2) The massless theory we consider is scale invariant at the classical level and, in general, there is a scale anomaly due to radiative corrections. The scale anomaly appears in the trace of the energy momentum tensor $T_{\mu \nu}$, which is traceless classically. It has the form

$$
T_{\mu}^{\mu}=\beta_{g} F^{\mu \nu} F_{\mu \nu}+\cdots
$$

(3) Massless, $N=1$ supersymmetric gauge theories are classically invariant under the supersymmetric extension of the conformal group - the superconformal group. Examining the superconformal algebra, it can be seen that the subset of superconformal transformations consisting of translations, SUSY transformations, and axial $R$ transformations is closed under SUSY, i.e. these transformations form a representation of SUSY. It follows that the conserved currents corresponding to these transformations make up a supermultiplet represented by an axial vector superfield called the supercurrent $J$,

$$
J \equiv\left\{J_{R}^{\prime \mu}, Q_{\alpha}^{\mu}, T_{\nu}^{\mu}, \ldots\right\}
$$

where $J_{R}^{\prime \mu}$ is the current associated to $\mathrm{R}$ invariance, $Q_{\alpha}^{\mu}$ is the one associated to SUSY invariance, and $T_{\nu}^{\mu}$ the one associated to translational invariance (energy-momentum tensor). 
The anomalies of the R current $J_{R}^{\prime \mu}$, the trace anomalies of the SUSY current, and the energy-momentum tensor, form also a second supermultiplet, called the supertrace anomaly

$$
\begin{aligned}
S= & \left\{\operatorname{Re} S, \operatorname{Im} S, S_{\alpha}\right\}= \\
& \left\{T_{\mu}^{\mu}, \partial_{\mu} J_{R}^{\prime \mu}, \sigma_{\alpha \dot{\beta}}^{\mu} \bar{Q}_{\mu}^{\dot{\beta}}+\cdots\right\}
\end{aligned}
$$

where $T_{\mu}^{\mu}$ in Eq.(35) and

$$
\begin{aligned}
\partial_{\mu} J_{R}^{\prime \mu} & =\beta_{g} \epsilon^{\mu \nu \sigma \rho} F_{\mu \nu} F_{\sigma \rho}+\cdots \\
\sigma_{\alpha \dot{\beta}}^{\mu} \bar{Q}_{\mu}^{\dot{\beta}} & =\beta_{g} \lambda^{\beta} \sigma_{\alpha \beta}^{\mu \nu} F_{\mu \nu}+\cdots
\end{aligned}
$$

(4) It is very important to note that the Noether current defined in (32) is not the same as the current associated to $\mathrm{R}$ invariance that appears in the supercurrent $J$ in (36), but they coincide in the tree approximation. So starting from a unique classical Noether current $J_{R(\text { class })}^{\mu}$, the Noether current $J_{R}^{\mu}$ is defined as the quantum extension of $J_{R(\text { class })}^{\mu}$ which allows for the validity of the non-renormalization theorem. On the other hand $J_{R}^{\prime \mu}$, is defined to belong to the supercurrent $J$, together with the energy-momentum tensor. The two requirements cannot be fulfilled by a single current operator at the same time.

Although the Noether current $J_{R}^{\mu}$ which obeys (33) and the current $J_{R}^{\prime \mu}$ belonging to the supercurrent multiplet $J$ are not the same, there is a relation [35, 36] between quantities associated with them

$$
r=\beta_{g}\left(1+x_{g}\right)+\beta_{i j k} x^{i j k}-\gamma_{A} r^{A}
$$

where $r$ was given in Eq. (34). The $r^{A}$ are the non-renormalized coefficients of the anomalies of the Noether currents associated to the chiral invariances of the superpotential, and like $r$ - are strictly one-loop quantities. The $\gamma_{A}$ 's are linear combinations of the anomalous dimensions of the matter fields, and $x_{g}$, and $x^{i j k}$ are radiative correction quantities. The structure of equality (40) is independent of the renormalization scheme.

One-loop finiteness, i.e. vanishing of the $\beta$-functions at one-loop, implies that the Yukawa couplings $\lambda_{i j k}$ must be functions of the gauge coupling $g$. To find a similar condition to all orders it is necessary and sufficient for the Yukawa couplings to be a formal power series in $g$, which is solution of the REs (301).

We can now state the theorem for all-order vanishing $\beta$-functions.

\section{Theorem:}

Consider an $N=1$ supersymmetric Yang-Mills theory, with simple gauge group. If the following conditions are satisfied

1. There is no gauge anomaly.

2. The gauge $\beta$-function vanishes at one-loop

$$
\beta_{g}^{(1)}=0=\sum_{i} l\left(R_{i}\right)-3 C_{2}(G) .
$$


3. There exist solutions of the form

$$
C_{i j k}=\rho_{i j k} g, \quad \rho_{i j k} \in \mathbb{C}
$$

to the conditions of vanishing one-loop matter fields anomalous dimensions

$$
\begin{aligned}
& \gamma_{j}^{i(1)}=0 \\
& =\frac{1}{32 \pi^{2}}\left[C^{i k l} C_{j k l}-2 g^{2} C_{2}\left(R_{i}\right) \delta_{i j}\right] .
\end{aligned}
$$

4. these solutions are isolated and non-degenerate when considered as solutions of vanishing one-loop Yukawa $\beta$-functions:

$$
\beta_{i j k}=0
$$

Then, each of the solutions (42) can be uniquely extended to a formal power series in $g$, and the associated super Yang-Mills models depend on the single coupling constant $g$ with a $\beta$ function which vanishes at all-orders.

It is important to note a few things: The requirement of isolated and non-degenerate solutions guarantees the existence of a unique formal power series solution to the reduction equations. The vanishing of the gauge $\beta$ function at one-loop, $\beta_{g}^{(1)}$, is equivalent to the vanishing of the $\mathrm{R}$ current anomaly (33). The vanishing of the anomalous dimensions at one-loop implies the vanishing of the Yukawa couplings $\beta$ functions at that order. It also implies the vanishing of the chiral anomaly coefficients $r^{A}$. This last property is a necessary condition for having $\beta$ functions vanishing at all orders 1 .

\section{Proof:}

Insert $\beta_{i j k}$ as given by the REs into the relationship (40) between the axial anomalies coefficients and the $\beta$-functions. Since these chiral anomalies vanish, we get for $\beta_{g}$ an homogeneous equation of the form

$$
0=\beta_{g}(1+O(\hbar)) .
$$

The solution of this equation in the sense of a formal power series in $\hbar$ is $\beta_{g}=0$, order by order. Therefore, due to the REs (30), $\beta_{i j k}=0$ too.

Thus we see that finiteness and reduction of couplings are intimately related. Since an equation like eq. (40) is lacking in non-supersymmetric theories, one cannot extend the validity of a similar theorem in such theories.

\subsection{Sum rule for SB terms in $N=1$ Supersymmetric and Finite theories: All-loop results}

As we have seen in Sect. 2.1, the method of reducing the dimensionless couplings has been extended [28], to the soft SUSY breaking (SSB) dimensionful parameters of $N=1$ supersymmetric theories. In addition it was found [60] that RGI SSB scalar masses in Gauge-Yukawa

\footnotetext{
${ }^{1}$ There is an alternative way to find finite theories [93].
} 
unified models satisfy a universal sum rule. Here we will describe first how the use of the available two-loop RG functions and the requirement of finiteness of the SSB parameters up to this order leads to the soft scalar-mass sum rule [61.

Consider the superpotential given by (20) along with the Lagrangian for SSB terms

$$
\begin{aligned}
-\mathcal{L}_{\mathrm{SB}} & =\frac{1}{6} h^{i j k} \phi_{i} \phi_{j} \phi_{k}+\frac{1}{2} b^{i j} \phi_{i} \phi_{j} \\
& +\frac{1}{2}\left(m^{2}\right)_{i}^{j} \phi^{* i} \phi_{j}+\frac{1}{2} M \lambda \lambda+\text { h.c. },
\end{aligned}
$$

where the $\phi_{i}$ are the scalar parts of the chiral superfields $\Phi_{i}, \lambda$ are the gauginos and $M$ their unified mass. Since we would like to consider only finite theories here, we assume that the gauge group is a simple group and the one-loop $\beta$-function of the gauge coupling $g$ vanishes. We also assume that the reduction equations admit power series solutions of the form

$$
C^{i j k}=g \sum_{n} \rho_{(n)}^{i j k} g^{2 n} .
$$

According to the finiteness theorem of Refs. [35, 36], the theory is then finite to all orders in perturbation theory, if, among others, the one-loop anomalous dimensions $\gamma_{i}^{j(1)}$ vanish. The one- and two-loop finiteness for $h^{i j k}$ can be achieved by [55]

$$
h^{i j k}=-M C^{i j k}+\cdots=-M \rho_{(0)}^{i j k} g+O\left(g^{5}\right),
$$

where ... stand for higher order terms.

Now, to obtain the two-loop sum rule for soft scalar masses, we assume that the lowest order coefficients $\rho_{(0)}^{i j k}$ and also $\left(m^{2}\right)_{j}^{i}$ satisfy the diagonality relations

$$
\rho_{i p q(0)} \rho_{(0)}^{j p q} \propto \delta_{i}^{j} \text { for all } p \text { and } q \text { and }\left(m^{2}\right)_{j}^{i}=m_{j}^{2} \delta_{j}^{i},
$$

respectively. Then we find the following soft scalar-mass sum rule [21,61, 94]

$$
\left(m_{i}^{2}+m_{j}^{2}+m_{k}^{2}\right) / M M^{\dagger}=1+\frac{g^{2}}{16 \pi^{2}} \Delta^{(2)}+O\left(g^{4}\right)
$$

for $\mathrm{i}, \mathrm{j}$, k with $\rho_{(0)}^{i j k} \neq 0$, where $\Delta^{(2)}$ is the two-loop correction

$$
\Delta^{(2)}=-2 \sum_{l}\left[\left(m_{l}^{2} / M M^{\dagger}\right)-(1 / 3)\right] T\left(R_{l}\right),
$$

which vanishes for the universal choice in accordance with the previous findings of Ref. [55.

If we know higher-loop $\beta$-functions explicitly, we can follow the same procedure and find higher-loop RGI relations among SSB terms. However, the $\beta$-functions of the soft scalar masses are explicitly known only up to two loops. In order to obtain higher-loop results some relations among $\beta$-functions are needed.

Making use of the spurion technique [49 53], it is possible to find the following all-loop relations among SSB $\beta$-functions, [41 46]

$$
\beta_{M}=2 \mathcal{O}\left(\frac{\beta_{g}}{g}\right)
$$




$$
\begin{aligned}
\beta_{h}^{i j k}= & \gamma^{i}{ }_{l} h^{l j k}+\gamma^{j}{ }_{l} h^{i l k}+\gamma^{k}{ }_{l} h^{i j l} \\
& -2 \gamma_{1 l}^{i} C^{l j k}-2 \gamma_{1 l}^{j} C^{i l k}-2 \gamma_{1 l}^{k} C^{i j l}, \\
\left(\beta_{m^{2}}\right)^{i}{ }_{j}= & {\left[\Delta+X \frac{\partial}{\partial g}\right] \gamma^{i}{ }_{j}, } \\
\mathcal{O}= & \left(M g^{2} \frac{\partial}{\partial g^{2}}-h^{l m n} \frac{\partial}{\partial C^{l m n}}\right), \\
\Delta= & 2 \mathcal{O} \mathcal{O}^{*}+2|M|^{2} g^{2} \frac{\partial}{\partial g^{2}}+\tilde{C}_{l m n} \frac{\partial}{\partial C_{l m n}}+\tilde{C}^{l m n} \frac{\partial}{\partial C^{l m n}},
\end{aligned}
$$

where $\left(\gamma_{1}\right)^{i}{ }_{j}=\mathcal{O} \gamma^{i}{ }_{j}, C_{l m n}=\left(C^{l m n}\right)^{*}$, and

$$
\tilde{C}^{i j k}=\left(m^{2}\right)^{i}{ }_{l} C^{l j k}+\left(m^{2}\right)^{j}{ }_{l} C^{i l k}+\left(m^{2}\right)^{k}{ }_{l} C^{i j l} .
$$

It was also found [42] that the relation

$$
h^{i j k}=-M\left(C^{i j k}\right)^{\prime} \equiv-M \frac{d C^{i j k}(g)}{d \ln g},
$$

among couplings is all-loop RGI. Furthermore, using the all-loop gauge $\beta$-function of Novikov et al. [62 64] given by

$$
\beta_{g}^{\mathrm{NSVZ}}=\frac{g^{3}}{16 \pi^{2}}\left[\frac{\sum_{l} T\left(R_{l}\right)\left(1-\gamma_{l} / 2\right)-3 C(G)}{1-g^{2} C(G) / 8 \pi^{2}}\right],
$$

it was found the all-loop RGI sum rule [47],

$$
\begin{aligned}
m_{i}^{2}+m_{j}^{2}+m_{k}^{2}= & |M|^{2}\left\{\frac{1}{1-g^{2} C(G) /\left(8 \pi^{2}\right)} \frac{d \ln C^{i j k}}{d \ln g}+\frac{1}{2} \frac{d^{2} \ln C^{i j k}}{d(\ln g)^{2}}\right\} \\
& +\sum_{l} \frac{m_{l}^{2} T\left(R_{l}\right)}{C(G)-8 \pi^{2} / g^{2}} \frac{d \ln C^{i j k}}{d \ln g} .
\end{aligned}
$$

In addition the exact- $\beta$-function for $m^{2}$ in the NSVZ scheme has been obtained [47] for the first time and is given by

$$
\begin{aligned}
\beta_{m_{i}^{2}}^{\mathrm{NSVZ}}= & {\left[|M|^{2}\left\{\frac{1}{1-g^{2} C(G) /\left(8 \pi^{2}\right)} \frac{d}{d \ln g}+\frac{1}{2} \frac{d^{2}}{d(\ln g)^{2}}\right\}\right.} \\
& \left.+\sum_{l} \frac{m_{l}^{2} T\left(R_{l}\right)}{C(G)-8 \pi^{2} / g^{2}} \frac{d}{d \ln g}\right] \gamma_{i}^{\mathrm{NSVZ}} .
\end{aligned}
$$

Surprisingly enough, the all-loop result (60) coincides with the superstring result for the finite case in a certain class of orbifold models [61] if $d \ln C^{i j k} / d \ln g=1$.

\section{$3 \quad$ Selecting the best model}

Finite Unified Theories (FUTs) have always attracted interest for their intriguing mathematical properties and their predictive power. One very important result is that the one-loop 
finiteness conditions (28,29) are sufficient to guarantee two-loop finiteness [74]. A classification of possible one-loop finite models was done by two groups [95 97]. The first one- and two-loop finite $S U(5)$ model was presented in [98], and shortly afterwards the conditions for finiteness in the soft SUSY-breaking sector at one-loop [79] were given. In [99] a one and two-loop finite $S U(5)$ model was presented where the rotation of the Higgs sector was proposed as a way of making it realistic. The first all-loop finite theory was studied in [23, 24, without taking into account the soft breaking terms. Finite soft breaking terms and the proof that one-loop finiteness in the soft terms also implies two-loop finiteness was done in [55]. The inclusion of soft breaking terms in a realistic model was done in [100] and their finiteness to all-loops studied in [45], although the universality of the soft breaking terms lead to a charged LSP. This fact was also noticed in [101], where the inclusion of an extra parameter in the boundary condition of the Higgs mixing mass parameter was introduced to alleviate it. The derivation of the sum-rule in the soft SUSY breaking sector and the proof that it can be made all-loop finite were done in Refs. [61] and [47] respectively, allowing thus for the construction of all-loop finite realistic models.

From the classification of theories with vanishing one-loop gauge $\beta$-function [95], one can easily see that there exist only two candidate possibilities to construct $S U(5)$ GUTs with three generations. These possibilities require that the theory should contain as matter fields the chiral supermultiplets $\mathbf{5}, \overline{\mathbf{5}}, \mathbf{1 0}, \overline{\mathbf{5}}, \mathbf{2 4}$ with the multiplicities $(6,9,4,1,0)$ or $(4,7,3,0,1)$, respectively. Only the second one contains a 24 -plet which can be used to provide the spontaneous symmetry breaking $(\mathrm{SB})$ of $S U(5)$ down to $S U(3) \times S U(2) \times U(1)$. For the first model one has to incorporate another way, such as the Wilson flux breaking mechanism in higher dimensional theories to achieve the desired SB of $S U(5)$ [23, 24]. Therefore, for a self-consistent field theory discussion we would like to concentrate only on the second possibility.

The particle content of the models we will study consists of the following supermultiplets: three $(\overline{\mathbf{5}}+\mathbf{1 0})$, needed for each of the three generations of quarks and leptons, four $(\overline{\mathbf{5}}+\mathbf{5})$ and one 24 considered as Higgs supermultiplets. When the gauge group of the finite GUT is broken the theory is no longer finite, and we will assume that we are left with the MSSM.

Therefore, a predictive Gauge-Yukawa unified $S U(5)$ model which is finite to all orders, in addition to the requirements mentioned already, should also have the following properties:

1. One-loop anomalous dimensions are diagonal, i.e., $\gamma_{i}^{(1) j} \propto \delta_{i}^{j}$.

2. The three fermion generations, in the irreducible representations $\overline{\mathbf{5}}_{i}, \mathbf{1 0}_{i}(i=1,2,3)$, should not couple to the adjoint 24.

3. The two Higgs doublets of the MSSM should mostly be made out of a pair of Higgs quintet and anti-quintet, which couple to the third generation.

In the following we discuss two versions of the all-order finite model: the model of Ref. [23, 24, which will be labeled A, and a slight variation of this model (labeled B), which can also be obtained from the class of the models suggested in Ref. 43, 44, with a modification to suppress non-diagonal anomalous dimensions [61. 


\begin{tabular}{|l|l|l|l|l|l|l|l|l|l|l|l|l|l|l|l|}
\hline & $\overline{\mathbf{5}}_{1}$ & $\overline{\mathbf{5}}_{2}$ & $\overline{\mathbf{5}}_{3}$ & $\mathbf{1 0}_{1}$ & $\mathbf{1 0}_{2}$ & $\mathbf{1 0}_{3}$ & $H_{1}$ & $H_{2}$ & $H_{3}$ & $H_{4}$ & $\bar{H}_{1}$ & $\bar{H}_{2}$ & $\bar{H}_{3}$ & $\bar{H}_{4}$ & $\mathbf{2 4}$ \\
\hline$Z_{7}$ & 4 & 1 & 2 & 1 & 2 & 4 & 5 & 3 & 6 & -5 & -3 & -6 & 0 & 0 & 0 \\
\hline$Z_{3}$ & 0 & 0 & 0 & 1 & 2 & 0 & 1 & 2 & 0 & -1 & -2 & 0 & 0 & 0 & 0 \\
\hline$Z_{2}$ & 1 & 1 & 1 & 1 & 1 & 1 & 0 & 0 & 0 & 0 & 0 & 0 & 0 & 0 & 0 \\
\hline
\end{tabular}

Table 1: Charges of the $Z_{7} \times Z_{3} \times Z_{2}$ symmetry for Model FUTA.

The superpotential which describes the two models before the reduction of couplings takes places is of the form [23, 24, 61, 98, 99]

$$
\begin{aligned}
W= & \sum_{i=1}^{3}\left[\frac{1}{2} g_{i}^{u} \mathbf{1 0}_{i} \mathbf{1 0}_{i} H_{i}+g_{i}^{d} \mathbf{1 0}_{i} \overline{\mathbf{5}}_{i} \bar{H}_{i}\right]+g_{23}^{u} \mathbf{1 0}_{2} \mathbf{1 0}_{3} H_{4} \\
& +g_{23}^{d} \mathbf{1 0}_{2} \overline{\mathbf{5}}_{3} \bar{H}_{4}+g_{32}^{d} \mathbf{1 0}_{3} \overline{\mathbf{5}}_{2} \bar{H}_{4}+\sum_{a=1}^{4} g_{a}^{f} H_{a} \mathbf{2 4} \bar{H}_{a}+\frac{g^{\lambda}}{3}(\mathbf{2 4})^{3},
\end{aligned}
$$

where $H_{a}$ and $\bar{H}_{a}(a=1, \ldots, 4)$ stand for the Higgs quintets and anti-quintets.

The main difference between model $\mathbf{A}$ and model $\mathbf{B}$ is that two pairs of Higgs quintets and anti-quintets couple to the $\mathbf{2 4} \mathrm{in} \mathbf{B}$, so that it is not necessary to mix them with $H_{4}$ and $\bar{H}_{4}$ in order to achieve the triplet-doublet splitting after the symmetry breaking of $S U(5)$ 61. Thus, although the particle content is the same, the solutions to Eqs. (26]27) and the sum rules are different, which will reflect in the phenomenology, as we will see.

\section{$3.1 \quad$ FUTA}

After the reduction of couplings the symmetry of the superpotential $W$ (62) is enhanced. For model $\mathbf{A}$ one finds that the superpotential has the $Z_{7} \times Z_{3} \times Z_{2}$ discrete symmetry with the charge assignment as shown in Table 1, and with the following superpotential

$$
W_{A}=\sum_{i=1}^{3}\left[\frac{1}{2} g_{i}^{u} \mathbf{1 0}_{i} \mathbf{1 0}_{i} H_{i}+g_{i}^{d} \mathbf{1 0}_{i} \overline{\mathbf{5}}_{i} \bar{H}_{i}\right]+g_{4}^{f} H_{4} \mathbf{2 4} \bar{H}_{4}+\frac{g^{\lambda}}{3}(\mathbf{2 4})^{3} .
$$

The non-degenerate and isolated solutions to $\gamma_{i}^{(1)}=0$ for model FUTA, which are the boundary conditions for the Yukawa couplings at the GUT scale, are:

$$
\begin{aligned}
& \left(g_{1}^{u}\right)^{2}=\frac{8}{5} g^{2},\left(g_{1}^{d}\right)^{2}=\frac{6}{5} g^{2},\left(g_{2}^{u}\right)^{2}=\left(g_{3}^{u}\right)^{2}=\frac{8}{5} g^{2}, \\
& \left(g^{\lambda}\right)^{2}=\frac{15}{7} g^{2},\left(g_{2}^{d}\right)^{2}=\left(g_{3}^{d}\right)^{2}=\frac{6}{5} g^{2},\left(g_{4}^{f}\right)^{2}=g^{2} \\
& \left(g_{23}^{u}\right)^{2}=\left(g_{23}^{d}\right)^{2}=\left(g_{32}^{d}\right)^{2}=\left(g_{2}^{f}\right)^{2}=\left(g_{3}^{f}\right)^{2}=\left(g_{1}^{f}\right)^{2}=0 .
\end{aligned}
$$

In the dimensionful sector, the sum rule gives us the following boundary conditions at the GUT scale for this model [61]:

$$
m_{H_{u}}^{2}+2 m_{\mathbf{1 0}}^{2}=m_{H_{d}}^{2}+m_{\overline{\mathbf{5}}}^{2}+m_{\mathbf{1 0}}^{2}=M^{2}
$$




\begin{tabular}{|l|l|l|l|l|l|l|l|l|l|l|l|l|l|l|l|}
\hline & $\overline{\mathbf{5}}_{1}$ & $\overline{\mathbf{5}}_{2}$ & $\overline{\mathbf{5}}_{3}$ & $\mathbf{1 0}_{1}$ & $\mathbf{1 0}_{2}$ & $\mathbf{1 0}_{3}$ & $H_{1}$ & $H_{2}$ & $H_{3}$ & $H_{4}$ & $\bar{H}_{1}$ & $\bar{H}_{2}$ & $\bar{H}_{3}$ & $\bar{H}_{4}$ & $\mathbf{2 4}$ \\
\hline$Z_{4}$ & 1 & 0 & 0 & 1 & 0 & 0 & 2 & 0 & 0 & 0 & -2 & 0 & 0 & 0 & 0 \\
\hline$Z_{4}$ & 0 & 1 & 0 & 0 & 1 & 0 & 0 & 2 & 0 & 3 & 0 & -2 & 0 & -3 & 0 \\
\hline$Z_{4}$ & 0 & 0 & 1 & 0 & 0 & 1 & 0 & 0 & 2 & 3 & 0 & 0 & -2 & -3 & 0 \\
\hline
\end{tabular}

Table 2: Charges of the $Z_{4} \times Z_{4} \times Z_{4}$ symmetry for Model FUTB.

and thus we are left with only three free parameters, namely $m_{\overline{\mathbf{5}}} \equiv m_{\overline{\mathbf{5}}_{3}}, m_{\mathbf{1 0}} \equiv m_{\mathbf{1 0}_{3}}$ and $M$.

\section{$3.2 \quad$ FUTB}

Also in the case of FUTB the symmetry is enhanced after the reduction of couplings. The superpotential has now a $Z_{4} \times Z_{4} \times Z_{4}$ symmetry with charges as shown in Table 2 and with the following superpotential

$$
\begin{aligned}
W_{B}=\sum_{i=1}^{3}[ & \left.\frac{1}{2} g_{i}^{u} \mathbf{1 0}_{i} \mathbf{1 0}_{i} H_{i}+g_{i}^{d} \mathbf{1} \mathbf{0}_{i} \overline{\mathbf{5}}_{i} \bar{H}_{i}\right]+g_{23}^{u} \mathbf{1 0}_{2} \mathbf{1 0}{ }_{3} H_{4} \\
& +g_{23}^{d} \mathbf{1 0}_{2} \overline{\mathbf{5}}_{3} \bar{H}_{4}+g_{32}^{d} \mathbf{1 0}_{3} \overline{\mathbf{5}}_{2} \bar{H}_{4}+g_{2}^{f} H_{2} \mathbf{2 4} \bar{H}_{2}+g_{3}^{f} H_{3} \mathbf{2 4} \bar{H}_{3}+\frac{g^{\lambda}}{3}(\mathbf{2 4})^{3},
\end{aligned}
$$

For this model the non-degenerate and isolated solutions to $\gamma_{i}^{(1)}=0$ give us:

$$
\begin{aligned}
& \left(g_{1}^{u}\right)^{2}=\frac{8}{5} g^{2},\left(g_{1}^{d}\right)^{2}=\frac{6}{5} g^{2},\left(g_{2}^{u}\right)^{2}=\left(g_{3}^{u}\right)^{2}=\left(g_{23}^{u}\right)^{2}=\frac{4}{5} g^{2}, \\
& \left(g_{2}^{d}\right)^{2}=\left(g_{3}^{d}\right)^{2}=\left(g_{23}^{d}\right)^{2}=\left(g_{32}^{d}\right)^{2}=\frac{3}{5} g^{2}, \\
& \left(g^{\lambda}\right)^{2}=\frac{15}{7} g^{2},\left(g_{2}^{f}\right)^{2}=\left(g_{3}^{f}\right)^{2}=\frac{1}{2} g^{2},\left(g_{1}^{f}\right)^{2}=\left(g_{4}^{f}\right)^{2}=0,
\end{aligned}
$$

and from the sum rule we obtain [61]:

$$
\begin{aligned}
m_{H_{u}}^{2}+2 m_{\mathbf{1 0}}^{2} & =M^{2}, m_{H_{d}}^{2}-2 m_{\mathbf{1 0}}^{2}=-\frac{M^{2}}{3} \\
m_{\overline{\mathbf{5}}}^{2}+3 m_{\mathbf{1 0}}^{2} & =\frac{4 M^{2}}{3},
\end{aligned}
$$

i.e., in this case we have only two free parameters $m_{\mathbf{1 0}} \equiv m_{\mathbf{1 0}_{3}}$ and $M$ for the dimensionful sector.

As already mentioned, after the $S U(5)$ gauge symmetry breaking we assume we have the MSSM, i.e. only two Higgs doublets. This can be achieved by introducing appropriate mass terms that allow to perform a rotation of the Higgs sector [23, 24, 98, 99, 102, in such a way that only one pair of Higgs doublets, coupled mostly to the third family, remains light and acquire vacuum expectation values. To avoid fast proton decay the usual fine tuning to 
achieve doublet-triplet splitting is performed. Notice that, although similar, the mechanism is not identical to minimal $S U(5)$, since we have an extended Higgs sector.

Thus, after the gauge symmetry of the GUT theory is broken we are left with the MSSM, with the boundary conditions for the third family given by the finiteness conditions, while the other two families are basically decoupled.

We will now examine the phenomenology of such all-loop Finite Unified theories with $S U(5)$ gauge group and, for the reasons expressed above, we will concentrate only on the third generation of quarks and leptons. An extension to three families, and the generation of quark mixing angles and masses in Finite Unified Theories has been addressed in [103], where several examples are given. These extensions are not considered here.

\subsection{Predictions for quark masses}

Since the gauge symmetry is spontaneously broken below $M_{\mathrm{GUT}}$, the finiteness conditions do not restrict the renormalization properties at low energies, and all it remains are boundary conditions on the gauge and Yukawa couplings (64) or (67), the $h=-M C$ relation (48), and the soft scalar-mass sum rule (50) at $M_{\mathrm{GUT}}$, as applied in the two models. Thus we examine the evolution of these parameters according to their RGEs up to two-loops for dimensionless parameters and at one-loop for dimensionful ones with the relevant boundary conditions. Below $M_{\text {GUT }}$ their evolution is assumed to be governed by the MSSM. We further assume a unique SUSY breaking scale $M_{\mathrm{SUSY}}$ (which we define as the geometrical average of the stop masses) and therefore below that scale the effective theory is just the SM. This allows to evaluate observables at or below the electroweak scale.

In the following, we review the prediction for the third generation of quark masses that allow for a direct comparison with experimental data and to determine the models that are in good agreement with the observed quark mass values. We discuss the current precision of the experimental results and the theoretical predictions. We also give relevant details of the higher-order perturbative corrections that we include, see Refs. [104 106] for more details. We do not discuss theoretical uncertainties from the RG running between the highscale parameters and the weak scale. At present, these uncertainties are expected to be less important than the experimental and theoretical uncertainties of the precision observables.

We now present the comparison of the predictions of the four models with the experimental data. In Fig. 1 we show the FUTA and FUTB predictions for the top pole mass, $M_{\text {top }}$, and the running bottom mass at the scale $M_{Z}, m_{\text {bot }}\left(M_{Z}\right)$, as a function of the unified gaugino mass $M$, for the two cases $\mu<0$ and $\mu>0$. The running bottom mass is used to avoid the large QCD uncertainties inherent to the pole mass. In the evaluation of the bottom mass $m_{\text {bot }}$, we have included the corrections coming from bottom squark-gluino loops and top squark-chargino loops [107]. We compare the predictions for the running bottom quark mass with the experimental value 108

$$
m_{b}\left(M_{Z}\right)=2.83 \pm 0.10 \mathrm{GeV} .
$$

One can see that the value of $m_{b}$ depends strongly on the sign of $\mu$ due to the above mentioned radiative corrections involving SUSY particles. For both models $\mathbf{A}$ and $\mathbf{B}$ the values for $\mu>0$ are above the central experimental value, with $m_{b}\left(M_{Z}\right) \sim 4.0-5.0 \mathrm{GeV}$. For $\mu<0$, on the other hand, model $\mathbf{B}$ shows overlap with the experimentally measured 
values, $m_{b}\left(M_{Z}\right) \sim 2.5-2.8 \mathrm{GeV}$. For model $\mathbf{A}$ we find $m_{b}\left(M_{Z}\right) \sim 1.5-2.6 \mathrm{GeV}$, and there is only a small region of allowed parameter space at large $M$ where we find agreement with the experimental value at the two $\sigma$ level. Therefore, the experimental determination of $m_{b}\left(M_{Z}\right)$ clearly selects the negative sign of $\mu$.

Now we turn to the top quark mass. The predictions for the top quark mass $m_{t}$ are $\sim 183$ and $\sim 172 \mathrm{GeV}$ in the models $\mathbf{A}$ and $\mathbf{B}$ respectively, as shown in the lower plot of fig. 1. Comparing these predictions with the experimental value [109]

$$
m_{t}^{\exp }=(173.2 \pm 0.9) \mathrm{GeV}
$$

and recalling that the theoretical values for $m_{t}$ may suffer from a correction of $\sim 4 \%$ [20, 94 , 110], we see that clearly model $\mathbf{B}$ is singled out. In addition the value of $\tan \beta$ is found to be $\tan \beta \sim 54$ and $\sim 48$ for models $\mathbf{A}$ and $\mathbf{B}$, respectively. Thus from the comparison of the predictions of the two models with experimental data only FUTB with $\mu<0$ survives.

\section{The best model before the Higgs discovery}

In the following we will concentrate on the model FUTB with $\mu<0$. We review the impact of further low-energy observables this model. As additional constraints we consider the following observables: the rare $b$ decays $\operatorname{BR}(b \rightarrow s \gamma)$ and $\operatorname{BR}\left(B_{s} \rightarrow \mu^{+} \mu^{-}\right)$.

For the branching ratio $\mathrm{BR}(b \rightarrow s \gamma)$, we take the value given by the Heavy Flavour Averaging Group (HFAG) is [11]

$$
\mathrm{BR}(b \rightarrow s \gamma)=\left(3.55 \pm 0.24_{-0.10}^{+0.09} \pm 0.03\right) \times 10^{-4}
$$

For the branching ratio $\mathrm{BR}\left(B_{s} \rightarrow \mu^{+} \mu^{-}\right)$, the $\mathrm{SM}$ prediction is at the level of $3 \times 10^{-9}$, in very good agreement with the recent $\mathrm{LHCb}$ measurement [112] experimental upper limit is

$$
\mathrm{BR}\left(B_{s} \rightarrow \mu^{+} \mu^{-}\right)=\left(3.2_{-1.2}^{+1.4}(\text { stat })_{-0.3}^{+0.5}(\text { syst })\right) \times 10^{-9}
$$

The final observable we include into the discussion is the cold dark matter (CDM) density. It is well known that the lightest neutralino, being the lightest supersymmetric particle (LSP), is an excellent candidate for CDM [113. Consequently one can demand that the lightest neutralino is indeed the LSP and parameters leading to a different LSP could be discarded.

The current bound, favored by a joint analysis of WMAP and other astrophysical and cosmological data, is at the $2 \sigma$ level given by the range [114],

$$
\Omega_{\mathrm{CDM}} h^{2}=0.1120 \pm 0.0112 \text {. }
$$

We find that no model point of FUTB with $\mu<0$ fulfills the strict bound of Eq. (73). Consequently, on a more general basis a mechanism is needed in our model to reduce the CDM abundance in the early universe. This issue could, for instance, be related to another problem, that of neutrino masses. This type of masses cannot be generated naturally within the class of finite unified theories that we are considering in this paper, although a non-zero value for neutrino masses has clearly been established [108]. However, the class of FUTs 

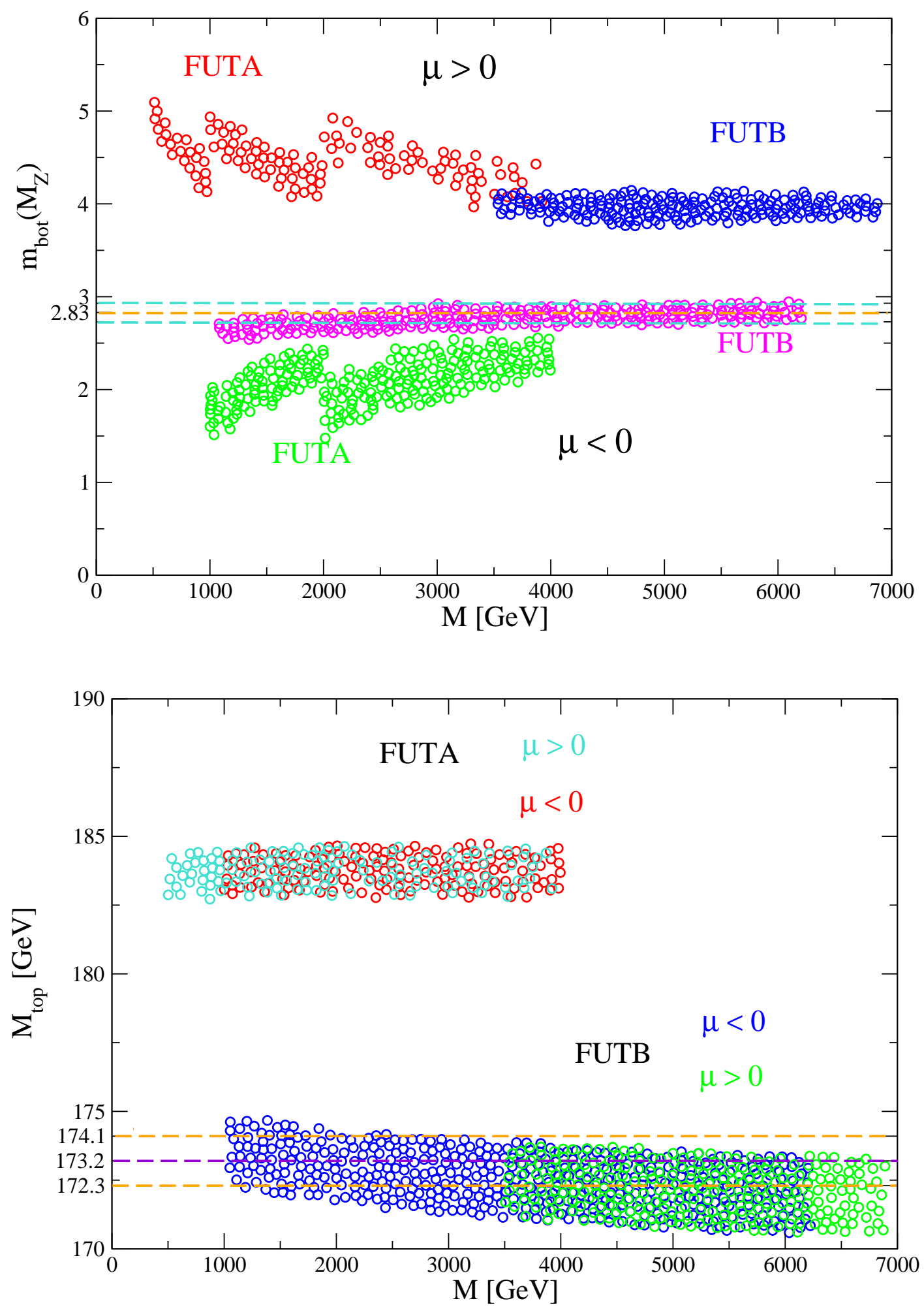

Figure 1: The bottom quark mass at the $Z$ boson scale (upper) and top quark pole mass (lower plot) are shown as function of $M$ for both models and both signs of $\mu$. 
discussed here can, in principle, be easily extended by introducing bilinear R-parity violating terms that preserve finiteness and introduce the desired neutrino masses [115]. R-parity violation [116] would have a small impact on the collider phenomenology presented here (apart from fact the SUSY search strategies could not rely on a 'missing energy' signature), but remove the CDM bound of Eq. (73) completely. The details of such a possibility in the present framework attempting to provide the models with realistic neutrino masses will be discussed elsewhere. Other mechanisms, not involving R-parity violation (and keeping the 'missing energy' signature), that could be invoked if the amount of CDM appears to be too large, concern the cosmology of the early universe. For instance, "thermal inflation" [117] or "late time entropy injection" [118] could bring the CDM density into agreement with the WMAP measurements. This kind of modifications of the physics scenario neither concerns the theory basis nor the collider phenomenology, but could have a strong impact on the CDM derived bounds.

Therefore, in order to get an impression of the possible impact of the CDM abundance on the collider phenomenology in our models under investigation, we will analyze the case that the LSP does contribute to the CDM density, and apply a more loose bound of

$$
\Omega_{\mathrm{CDM}} h^{2}<0.3 .
$$

(Lower values than the ones permitted by Eq. (73) are naturally allowed if another particle than the lightest neutralino constitutes CDM.) For our evaluation we have used the code MicroMegas [119.

Taking these observables into account we predict the light $\mathcal{C} \mathcal{P}$-even Higgs boson mass of the model. For the mass prediction we use the code FeynHiggs [120 124]. The prediction for $M_{h}$ of FUTB with $\mu<0$ is shown in Fig. 2. All points shown are in agreement with the constraints from the two $B$ physics observables as described above. The dark (red) points are also in agreement with the loose CDM bound described above. The lightest Higgs mass ranges in

$$
M_{h} \sim 121-126 \mathrm{GeV},
$$

where the uncertainty comes from variations of the soft scalar masses. To this value one has to add at least $\pm 2 \mathrm{GeV}$ coming from unkonwn higher order corrections [122]. We have also included a small variation, due to threshold corrections at the GUT scale, of up to $5 \%$ of the FUT boundary conditions. The masses of the heavier Higgs bosons are found at

relatively high values, above the $\mathrm{TeV}$ scale (see also the next subsection). This is due to the relatively stringent bound on $\operatorname{BR}\left(B_{s} \rightarrow \mu^{+} \mu^{-}\right)$, which pushes the heavy Higgs masses beyond $\sim 1 \mathrm{TeV}$, excluding their discovery at the LHC.

As further features a generally heavy SUSY spectrum and large values of $\tan \beta$ (the ratio of the two vacuum expectation values) were found [104]. We furthermore find in our analysis that the lightest observable SUSY particle (LOSP) is always the light scalar tau, with its mass starting around $\sim 500 \mathrm{GeV}$.

\section{The best model after the Higgs discovery}

The spectacular discovery of a Higgs boson at ATLAS and CMS, as announced in July 2012 [65, 66] can be interpreted as the discovery of the light $\mathcal{C P}$-even Higgs boson of the 


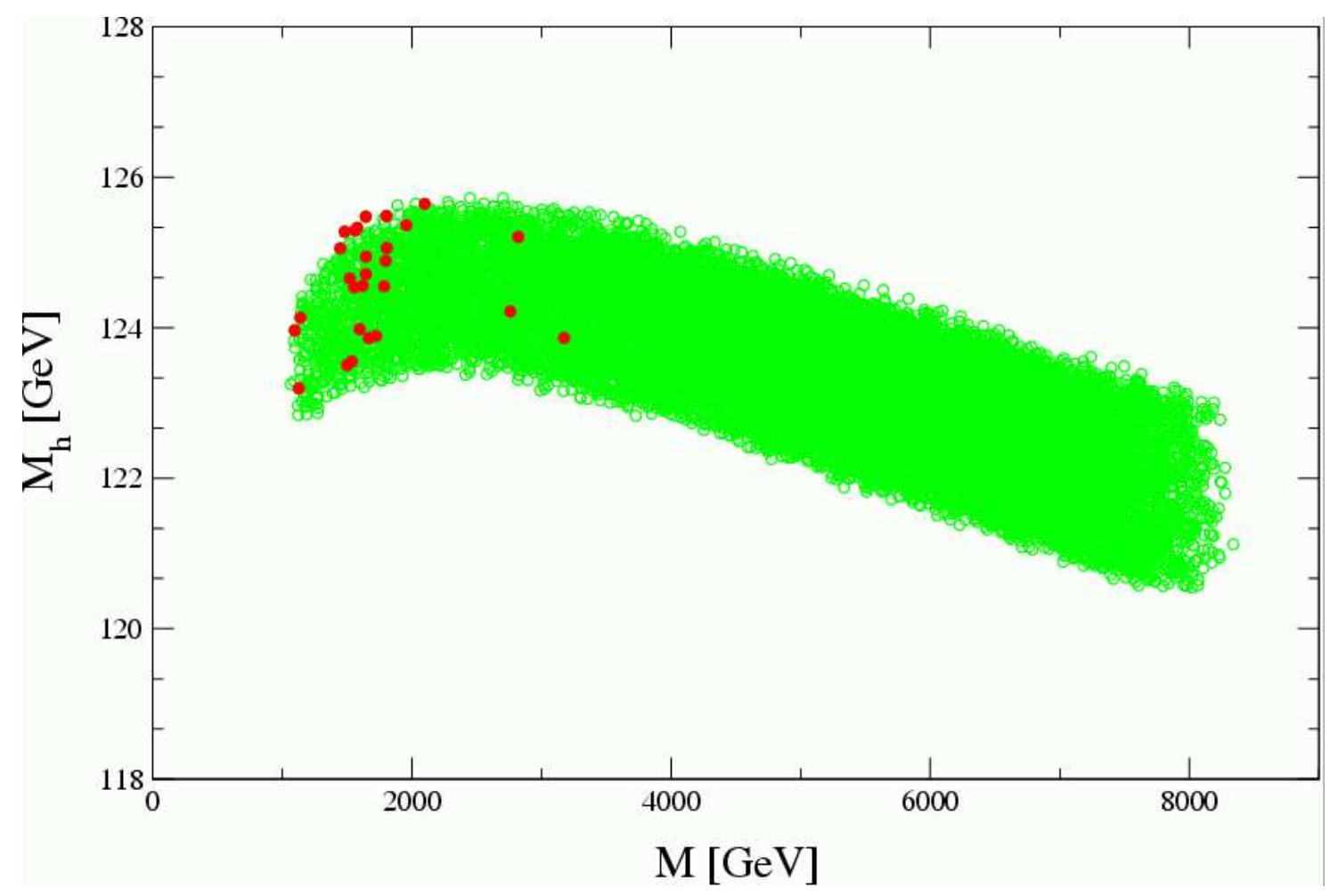

Figure 2: The lightest Higgs boson mass, $M_{h}$, as a function of $M$ in the model FUTB with $\mu<0$. All points shown fulfill the $B$-physics constraints (see text). The dark (red) points in addition are in agreement with the (lose) CDM constraint (see text).

MSSM Higgs spectrum [125[129]. Consequently, as a crucial new ingredient we take into account the recent discovery of a Higgs boson with a mass measurement of

$$
M_{h} \sim 126.0 \pm 1 \pm 2 \mathrm{GeV},
$$

where \pm 1 comes from the experimental error and \pm 2 corresponds to the theoretical error, and see how this affects the SUSY spectrum. Constraining the allowed values of the Higgs mass this way puts a limit on the allowed values of the unified gaugino mass, as can be seen from Fig. 3. The red lines correspond to the pure experimental uncertainty and restrict $2 \mathrm{TeV} \lesssim M \lesssim 5 \mathrm{TeV}$, the blue line includes the additional theory uncertainty of $\pm 2 \mathrm{GeV}$. Taking this uncertainty into account no bound on $M$ can be placed. However, a substantial part of the formerly allowed parameter points are now excluded. This in turn restricts the LOSP, which continues to be the light scalar tau. In Fig. [4 the effects on the mass of the LOSP are demonstrated. Without any Higgs mass constraint all coloured points are allowed. Imposing $M_{h}=126 \pm 1 \mathrm{GeV}$ only the green (light shaded) points are allowed, restricting the mass to be between about $500 \mathrm{GeV}$ and $2500 \mathrm{GeV}$, the lower values might be experimentally accessible at the ILC with $1000 \mathrm{GeV}$ center-of-mass energy or at CLIC with an energy up to $\sim 3 \mathrm{TeV}$. Taking into account the theory uncertainty on $M_{h}$ also the blue (dark shaded) points are allowed, permitting the LOSP mass up to $\sim 4 \mathrm{TeV}$. If the upper end of the parameter space were realized the light scalar tau would remain unobservable even at CLIC. 


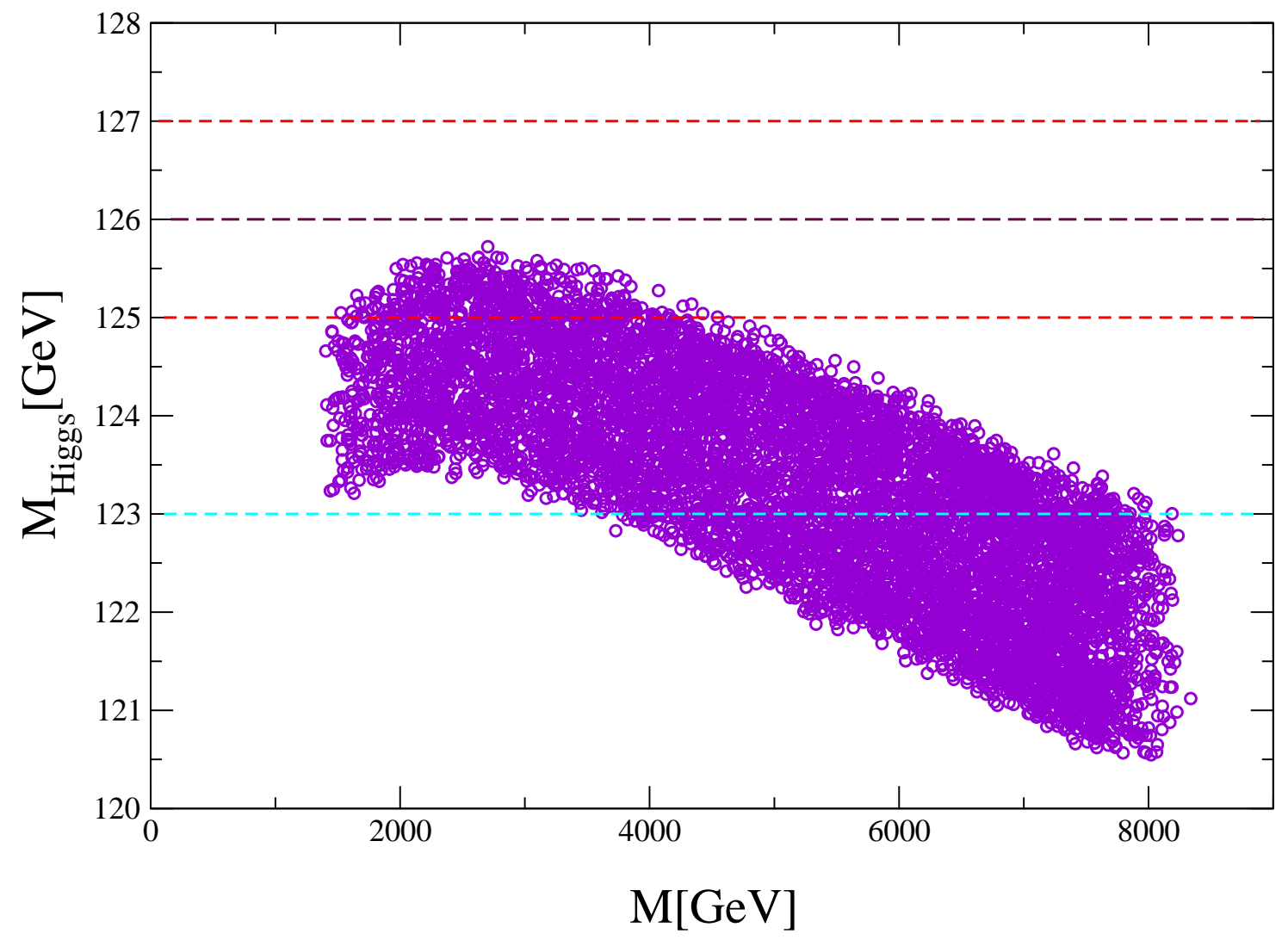

Figure 3: The lightest Higgs boson mass, $M_{h}$, as a function of $M$ in the model FUTB with $\mu<0$. All points shown fulfill the $B$-physics constraints (see text).

The full particle spectrum of model FUTB with $\mu<0$, compliant with quark mass constraints and the $B$-physics observables is shown in Fig. 5, In the upper (lower) plot we impose $M_{h}=126 \pm 3(1) \mathrm{GeV}$. Without any $M_{h}$ restrictions the coloured SUSY particles have masses above $\sim 1.8 \mathrm{TeV}$ in agreement with the non-observation of those particles at the LHC [130-132]. Including the Higgs mass constraints in general favours the lower part of the SUSY particle mass spectra, but also cuts away the very low values. Neglecting the theory uncertainties of $M_{h}$ (as shown in the lower plot of Fig. 5) permits SUSY masses which would remain unobservable at the LHC, the ILC or CLIC. On the other hand, large parts of the allowed spectrum of the lighter scalar tau or the lighter neutralinos might be accessible at CLIC with $\sqrt{s}=3 \mathrm{TeV}$. Including the theory uncertainties, even higher masses are permitted, further weakening the discovery potential of the LHC and future $e^{+} e^{-}$colliders. A numerical example of the lighter part of the spectrum is shown in Table 3. If such a spectrum were realized, the coloured particles are at the border of the discovery region at the LHC. Some uncoloured particles like the scalar taus, the light chargino or the lighter neutralinos would be in the reach of a high-energy Linear Collider.

Finally, we note that with such a heavy SUSY spectrum the anomalous magnetic moment of the muon, $(g-2)_{\mu}$ (with $\left.a_{\mu} \equiv(g-2)_{\mu} / 2\right)$, gives only a negligible correction to the SM prediction. The comparison of the experimental result and the SM value (based on the latest 


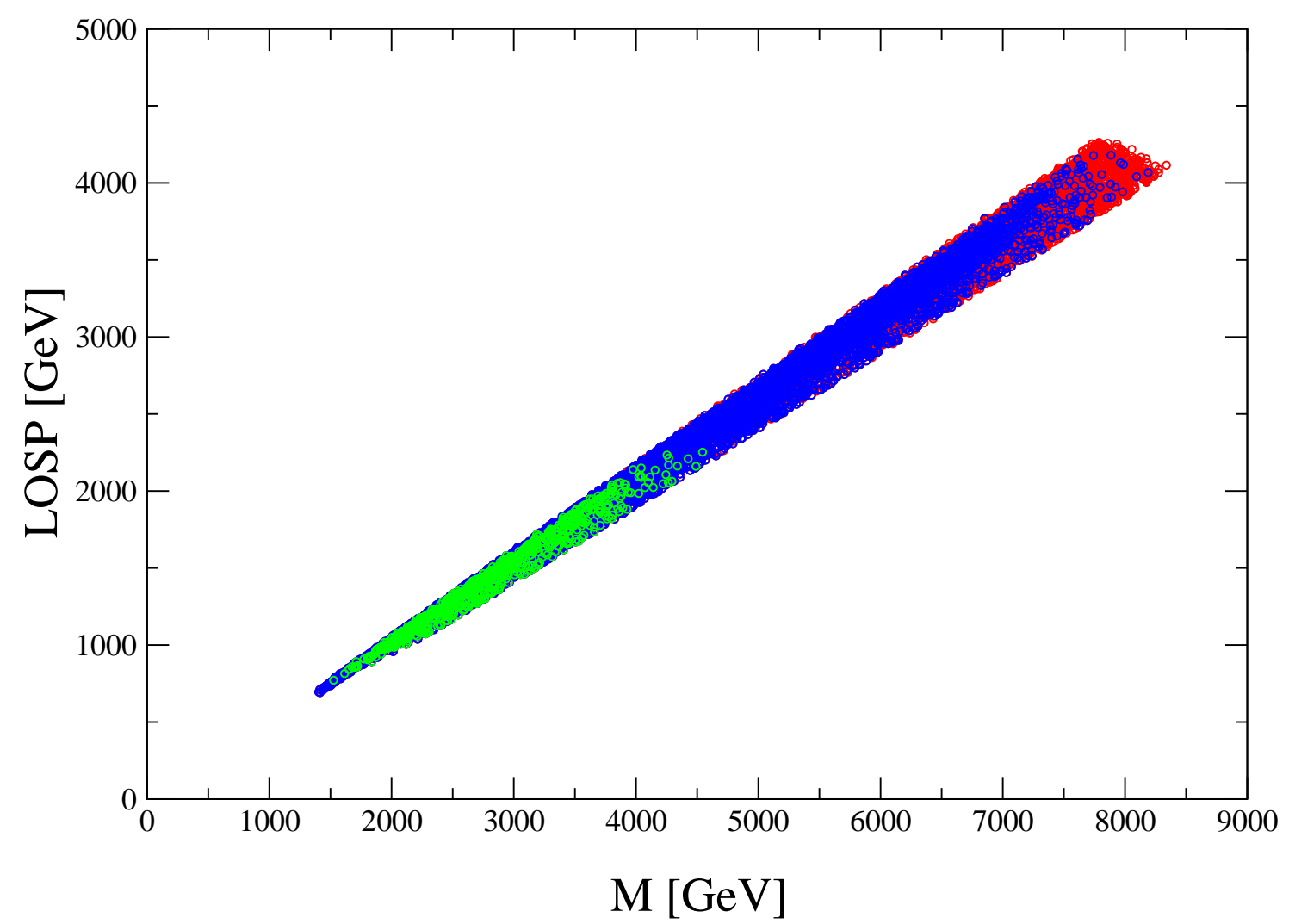

Figure 4: The mass of the LOSP (the light scalar tau) is presented as a function of $M$. Shown are only points that fulfill the $B$ physics constraints. The green (light shaded) points correspond to $M_{h}=126 \pm 1 \mathrm{GeV}$, the blue (dark shaded) points have $M_{h}=126 \pm 3 \mathrm{GeV}$, and the red points have no $M_{h}$ restriction.

combination using $e^{+} e^{-}$data) [133]

$$
a_{\mu}^{\exp }-a_{\mu}^{\mathrm{SM}}=(28.7 \pm 8.0) \times 10^{-10},
$$

would disfavor FUTB with $\mu<0$ [134,135]. However, since the results would be very close to the SM results, the model has the same level of difficulty with the $a_{\mu}$ measurement as the SM.

\section{Conclusions}

A number of proposals and ideas have matured with time and have survived after careful theoretical studies and confrontation with experimental data. These include part of the original GUTs ideas, mainly the unification of gauge couplings and, separately, the unification of the Yukawa couplings, a version of fixed point behaviour of couplings, and certainly the necessity of SUSY as a way to take care of the technical part of the hierarchy problem. On the other hand, a very serious theoretical problem, namely the presence of divergencies in Quantum Field Theories (QFT), although challenged by the founders of QFT [136 138, 


\begin{tabular}{|l|l||l|l|}
\hline$m_{b}\left(M_{Z}\right)$ & 2.74 & $m_{t}$ & 174.1 \\
\hline$M_{h}$ & 125.0 & $M_{A}$ & 1517 \\
\hline$M_{H}$ & 1515 & $M_{H^{ \pm}}$ & 1518 \\
\hline$m_{\tilde{t}_{1}}$ & 2483 & $m_{\tilde{t}_{2}}$ & 2808 \\
\hline$m_{\tilde{b}_{1}}$ & 2403 & $m_{\tilde{b}_{2}}$ & 2786 \\
\hline$m_{\tilde{\tau}_{1}}$ & 892 & $m_{\tilde{\tau}_{2}}$ & 1089 \\
\hline$m_{\tilde{\chi}_{1}^{ \pm}}$ & 1453 & $m_{\tilde{\chi}_{2}^{ \pm}}$ & 2127 \\
\hline$m_{\tilde{\chi}_{1}^{0}}$ & 790 & $m_{\tilde{\chi}_{2}^{0}}$ & 1453 \\
\hline$m_{\tilde{\chi}_{3}^{0}}$ & 2123 & $m_{\tilde{\chi}_{4}^{0}}$ & 2127 \\
\hline$m_{\tilde{g}}$ & 3632 & & \\
\hline
\end{tabular}

Table 3: A representative spectrum of a light FUTB, $\mu<0$ spectrum, compliant with the $B$ physics constraints. All masses are in $\mathrm{GeV}$.

was mostly forgotten in the course of developments of the field partly due to the spectacular successes of renormalizable field theories, in particular of the SM. However, as it was already mentioned in the Introduction, fundamental developments in theoretical particle physics are based in reconsiderations of the problem of divergencies and serious attempts to solve it. These include the motivation and construction of string and non-commutative theories, as well as $N=4$ supersymmetric field theories [3, 4], $N=8$ supergravity [5] and the AdS/CFT correspondence [2]. It is a thoroughly fascinating fact that many interesting ideas that have survived various theoretical and phenomenological tests, as well as the solution to the UV divergencies problem, find a common ground in the framework of $N=1$ Finite Unified Theories, which we have described in the previous sections. From the theoretical side they solve the problem of UV divergencies in a minimal way. On the phenomenological side, since they are based on the principle of reduction of couplings (expressed via RGI relations among couplings and masses), they provide strict selection rules in choosing realistic models which lead to testable predictions.

We examined the predictions of two $S U(5)$ Finite Unified Theories in light of the new bounds on the branching ratio $\mathrm{BR}\left(B_{s} \rightarrow \mu^{+} \mu^{-}\right)$. Only one model, FUTB with $\mu<0$, is consistent with all the phenomenological constraints. Compared to our previous analysis [104, the new bound on $\operatorname{BR}\left(B_{s} \rightarrow \mu^{+} \mu^{-}\right)$excludes values for the heavy Higgs bosons masses below $1 \sim \mathrm{TeV}$, and in general allows only a very heavy SUSY spectrum. The Higgs mass constraint favours the lower part of this spectrum, with SUSY masses ranging from $\sim 500 \mathrm{GeV}$ up to the multi-TeV level, where the lower part of the spectrum could be accessible at the ILC or CLIC.

The celebrated success of predicting the top-quark mass [23 28] has been extended to the predictions of the Higgs masses and the supersymmetric spectrum of the MSSM [104, 139. The predicted mass of the lightest Higgs boson turns out to be naturally in agreement with the discovery of a Higgs-like state at the LHC. Identifying the lightest Higgs boson with the newly discovered state we restricted the allowed parameter space of the FUTB with $\mu<0$. We reviewed how this reduction of parameter space impacts the prediction of the SUSY spectrum. Overall, the resulting spectrum turns out to be heavy, mostly outside the reach of the LHC, ILC or even CLIC. A light Higgs boson with characteristics very close to a SM 

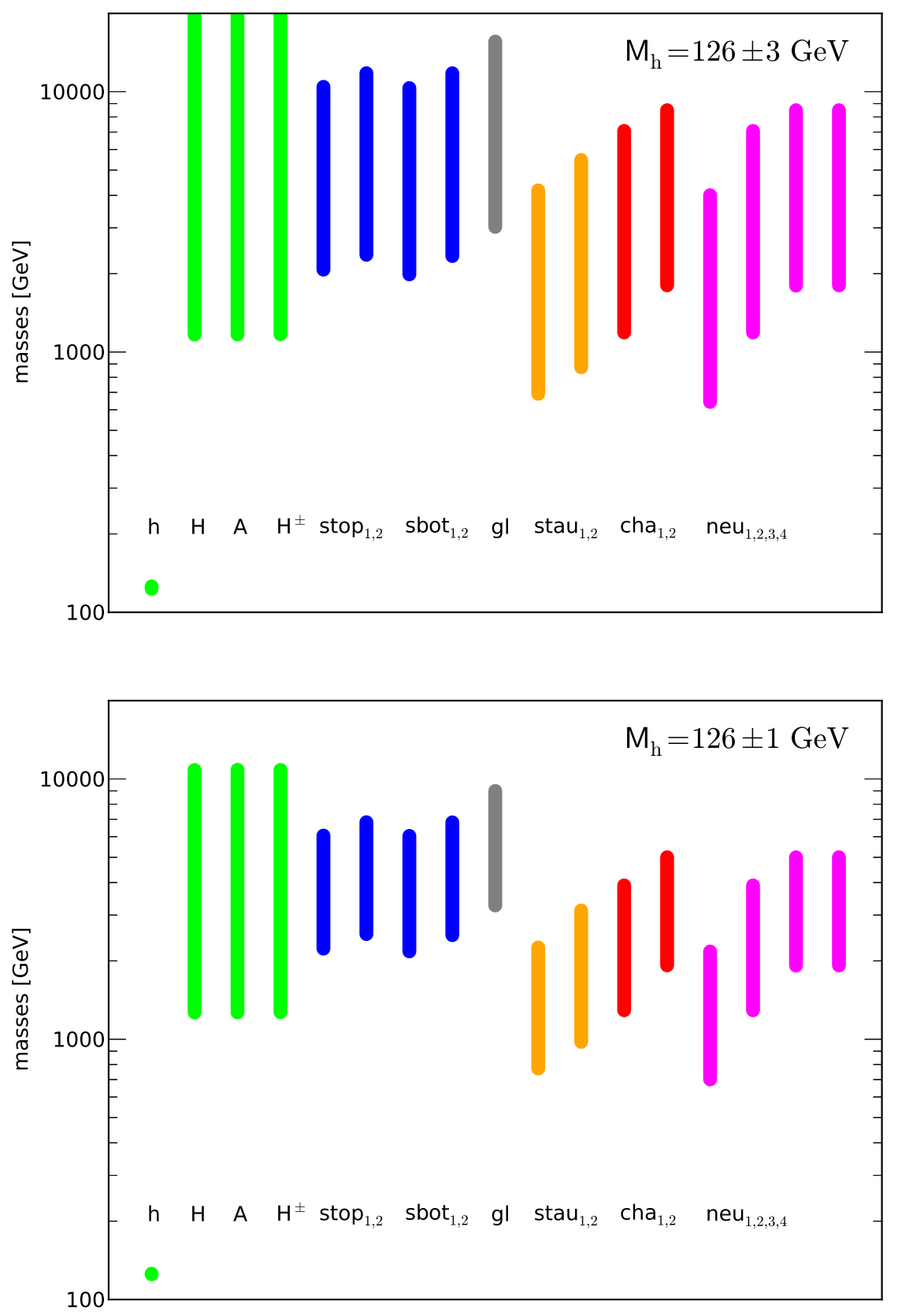

Figure 5: The upper (lower) plot shows the spectrum after imposing the constraint $M_{h}=$ $126 \pm 3(1) \mathrm{GeV}$. The particle spectrum of model FUTB with $\mu<0$, where the points shown are in agreement with the quark mass constraints and the $B$-physics observables. The light (green) points on the left are the various Higgs boson masses. The dark (blue) points following are the two scalar top and bottom masses, followed by the lighter (gray) gluino mass. Next come the lighter (beige) scalar tau masses. The darker (red) points to the right are the two chargino masses followed by the lighter shaded (pink) points indicating the neutralino masses. 
Higgs boson could remain as the only possible experimental observation.

\section{Acknowledgements}

We acknowledge useful discussions with W. Hollik, C. Kounnas, D. Lüst, C. Muñoz, and W. Zimmermann. The work of G.Z. is supported by the European Unions ITN programme "UNILHC" and the Research Funding Program "ARISTEIA", "Higher Order Calculations and Tools for High Energy Colliders", HOCTools (co-financed by the European Union (European Social Fund ESF) and Greek national funds through the Operational Program "Education and Lifelong Learning" of the National Strategic Reference Framework (NSRF)). The work of S.H. was supported in part by CICYT (grant FPA 2010-22163-C02-01) and by the Spanish MICINN's Consolider-Ingenio 2010 Program under grant MultiDark CSD2009-

00064. The work of M.M. was supported in part by mexican grants PAPIIT IN113712 and Conacyt 132059. 


\section{References}

[1] A. Connes, M. R. Douglas and A. S. Schwarz, JHEP 02, 003 (1998), hep-th/9711162.

[2] J. M. Maldacena, Adv. Theor. Math. Phys. 2, 231 (1998), hep-th/9711200.

[3] S. Mandelstam, Nucl. Phys. B213, 149 (1983).

[4] L. Brink, O. Lindgren and B. E. W. Nilsson, Phys. Lett. B123, 323 (1983).

[5] Z. Bern, J. J. Carrasco, L. J. Dixon, H. Johansson and R. Roiban, Phys. Rev. Lett. 103, 081301 (2009), [0905.2326].

[6] R. Kallosh, JHEP 09, 116 (2009), [0906.3495].

[7] Z. Bern et al., Phys. Rev. Lett. 98, 161303 (2007), hep-th/0702112.

[8] Z. Bern, L. J. Dixon and R. Roiban, Phys. Lett. B644, 265 (2007), hep-th/0611086].

[9] M. B. Green, J. G. Russo and P. Vanhove, Phys. Rev. Lett. 98, 131602 (2007), hep-th/0611273.

[10] J. C. Pati and A. Salam, Phys. Rev. Lett. 31, 661 (1973).

[11] H. Georgi and S. L. Glashow, Phys. Rev. Lett. 32, 438 (1974).

[12] H. Georgi, H. R. Quinn and S. Weinberg, Phys. Rev. Lett. 33, 451 (1974).

[13] H. Fritzsch and P. Minkowski, Ann. Phys. 93, 193 (1975).

[14] H. Georgi, Particles and Fields: Williamsburg 1974. AIP Conference Proceedings No. 23, American Institute of Physics, New York, 1974, ed. Carlson, C. E.

[15] U. Amaldi, W. de Boer and H. Furstenau, Phys. Lett. B260, 447 (1991).

[16] S. Dimopoulos and H. Georgi, Nucl. Phys. B193, 150 (1981).

[17] N. Sakai, Zeit. Phys. C11, 153 (1981).

[18] A. J. Buras, J. R. Ellis, M. K. Gaillard and D. V. Nanopoulos, Nucl. Phys. B135, 66 (1978).

[19] J. Kubo, M. Mondragon, M. Olechowski and G. Zoupanos, Nucl. Phys. B479, 25 (1996), hep-ph/9512435.

[20] J. Kubo, M. Mondragon and G. Zoupanos, Acta Phys. Polon. B27, 3911 (1997), hep-ph/9703289.

[21] T. Kobayashi, J. Kubo, M. Mondragon and G. Zoupanos, Acta Phys. Polon. B30, 2013 (1999).

[22] P. Fayet, Nucl. Phys. B149, 137 (1979). 
[23] D. Kapetanakis, M. Mondragon and G. Zoupanos, Z. Phys. C60, 181 (1993), hep-ph/9210218.

[24] M. Mondragon and G. Zoupanos, Nucl. Phys. Proc. Suppl. 37C, 98 (1995).

[25] J. Kubo, M. Mondragon and G. Zoupanos, Nucl. Phys. B424, 291 (1994).

[26] J. Kubo, M. Mondragon, N. D. Tracas and G. Zoupanos, Phys. Lett. B342, 155 (1995), hep-th/9409003.

[27] J. Kubo, M. Mondragon, S. Shoda and G. Zoupanos, Nucl. Phys. B469, 3 (1996), hep-ph/9512258.

[28] J. Kubo, M. Mondragon and G. Zoupanos, Phys. Lett. B389, 523 (1996), hep-ph/9609218.

[29] W. Zimmermann, Commun. Math. Phys. 97, 211 (1985).

[30] R. Oehme and W. Zimmermann, Commun. Math. Phys. 97, 569 (1985).

[31] E. Ma, Phys. Rev. D17, 623 (1978).

[32] E. Ma, Phys. Rev. D31, 1143 (1985).

[33] N. -P. Chang, Phys. Rev. D 10 (1974) 2706.

[34] S. Nandi and W. -C. Ng, Phys. Rev. D 20 (1979) 972.

[35] C. Lucchesi, O. Piguet and K. Sibold, Phys. Lett. B201, 241 (1988).

[36] C. Lucchesi, O. Piguet and K. Sibold, Helv. Phys. Acta 61, 321 (1988).

[37] C. Lucchesi and G. Zoupanos, Fortschr. Phys. 45, 129 (1997), hep-ph/9604216].

[38] A. V. Ermushev, D. I. Kazakov and O. V. Tarasov, Nucl. Phys. B281, 72 (1987).

[39] D. I. Kazakov, Mod. Phys. Lett. A2, 663 (1987).

[40] I. Jack and D. R. T. Jones, Phys. Lett. B349, 294 (1995), hep-ph/9501395].

[41] J. Hisano and M. A. Shifman, Phys. Rev. D56, 5475 (1997), hep-ph/9705417.

[42] I. Jack and D. R. T. Jones, Phys. Lett. B415, 383 (1997), hep-ph/9709364.

[43] L. V. Avdeev, D. I. Kazakov and I. N. Kondrashuk, Nucl. Phys. B510, 289 (1998), hep-ph/9709397.

[44] D. I. Kazakov, Phys. Lett. B449, 201 (1999), hep-ph/9812513.

[45] D. I. Kazakov, Phys. Lett. B421, 211 (1998), hep-ph/9709465.

[46] I. Jack, D. R. T. Jones and A. Pickering, Phys. Lett. B426, 73 (1998), hep-ph/9712542. 
[47] T. Kobayashi, J. Kubo and G. Zoupanos, Phys. Lett. B427, 291 (1998), hep-ph/9802267.

[48] Y. Yamada, Phys. Rev. D50, 3537 (1994), hep-ph/9401241.

[49] R. Delbourgo, Nuovo Cim. A25, 646 (1975).

[50] A. Salam and J. A. Strathdee, Nucl. Phys. B86, 142 (1975).

[51] K. Fujikawa and W. Lang, Nucl. Phys. B88, 61 (1975).

[52] M. T. Grisaru, W. Siegel and M. Rocek, Nucl. Phys. B159, 429 (1979).

[53] L. Girardello and M. T. Grisaru, Nucl. Phys. B194, 65 (1982).

[54] D. R. T. Jones, L. Mezincescu and Y. P. Yao, Phys. Lett. B148, 317 (1984).

[55] I. Jack and D. R. T. Jones, Phys. Lett. B333, 372 (1994), hep-ph/9405233.

[56] L. E. Ibanez and D. Lust, Nucl. Phys. B382, 305 (1992), hep-th/9202046.

[57] V. S. Kaplunovsky and J. Louis, Phys. Lett. B306, 269 (1993), hep-th/9303040.

[58] A. Brignole, L. E. Ibanez and C. Munoz, Nucl. Phys. B422, 125 (1994), hep-ph/9308271.

[59] J. A. Casas, A. Lleyda and C. Munoz, Phys. Lett. B380, 59 (1996), hep-ph/9601357].

[60] Y. Kawamura, T. Kobayashi and J. Kubo, Phys. Lett. B405, 64 (1997), hep-ph/9703320.

[61] T. Kobayashi, J. Kubo, M. Mondragon and G. Zoupanos, Nucl. Phys. B511, 45 (1998), hep-ph/9707425].

[62] V. A. Novikov, M. A. Shifman, A. I. Vainshtein and V. I. Zakharov, Nucl. Phys. B229, 407 (1983).

[63] V. A. Novikov, M. A. Shifman, A. I. Vainshtein and V. I. Zakharov, Phys. Lett. B166, 329 (1986).

[64] M. A. Shifman, Int. J. Mod. Phys. A11, 5761 (1996), hep-ph/9606281.

[65] ATLAS Collaboration, G. Aad et al., Phys.Lett.B (2012), [1207.7214].

[66] CMS Collaboration, S. Chatrchyan et al., Phys.Lett.B (2012), [1207.7235].

[67] R. Oehme, Prog. Theor. Phys. Suppl. 86, 215 (1986).

[68] J. Kubo, K. Sibold and W. Zimmermann, Nucl. Phys. B259, 331 (1985).

[69] J. Kubo, K. Sibold and W. Zimmermann, Phys. Lett. B220, 185 (1989). 
[70] O. Piguet and K. Sibold, Phys. Lett. B229, 83 (1989).

[71] W. Zimmermann, Lect. Notes Phys. 539, 304 (2000).

[72] J. Wess and B. Zumino, Phys. Lett. B49, 52 (1974).

[73] J. Iliopoulos and B. Zumino, Nucl. Phys. B76, 310 (1974).

[74] A. Parkes and P. C. West, Phys. Lett. B138, 99 (1984).

[75] S. Rajpoot and J. G. Taylor, Phys. Lett. B147, 91 (1984).

[76] S. Rajpoot and J. G. Taylor, Int. J. Theor. Phys. 25, 117 (1986).

[77] P. C. West, Phys. Lett. B137, 371 (1984).

[78] D. R. T. Jones and A. J. Parkes, Phys. Lett. B160, 267 (1985).

[79] D. R. T. Jones and L. Mezincescu, Phys. Lett. B138, 293 (1984).

[80] A. J. Parkes, Phys. Lett. B156, 73 (1985).

[81] L. O’Raifeartaigh, Nucl. Phys. B96, 331 (1975).

[82] P. Fayet and J. Iliopoulos, Phys. Lett. B51, 461 (1974).

[83] S. Ferrara and B. Zumino, Nucl. Phys. B87, 207 (1975).

[84] O. Piguet and K. Sibold, Nucl. Phys. B196, 428 (1982).

[85] O. Piguet and K. Sibold, Nucl. Phys. B196, 447 (1982).

[86] O. Piguet and K. Sibold, Int. J. Mod. Phys. A1, 913 (1986).

[87] O. Piguet and K. Sibold, Phys. Lett. B177, 373 (1986).

[88] P. Ensign and K. T. Mahanthappa, Phys. Rev. D36, 3148 (1987).

[89] O. Piguet, hep-th/9606045, Talk given at 10th International Conference on Problems of Quantum Field Theory.

[90] L. Alvarez-Gaume and P. H. Ginsparg, Nucl. Phys. B243, 449 (1984).

[91] W. A. Bardeen and B. Zumino, Nucl. Phys. B244, 421 (1984).

[92] B. Zumino, Y.-S. Wu and A. Zee, Nucl. Phys. B239, 477 (1984).

[93] R. G. Leigh and M. J. Strassler, Nucl. Phys. B447, 95 (1995), hep-th/9503121.

[94] M. Mondragon and G. Zoupanos, Acta Phys. Polon. B34, 5459 (2003).

[95] S. Hamidi, J. Patera and J. H. Schwarz, Phys. Lett. B141, 349 (1984). 
[96] X.-D. Jiang and X.-J. Zhou, Phys. Lett. B216, 160 (1989).

[97] X.-d. Jiang and X.-j. Zhou, Phys. Lett. B197, 156 (1987).

[98] D. R. T. Jones and S. Raby, Phys. Lett. B143, 137 (1984).

[99] J. Leon, J. Perez-Mercader, M. Quiros and J. Ramirez-Mittelbrunn, Phys. Lett. B156, 66 (1985).

[100] D. I. Kazakov, M. Y. Kalmykov, I. N. Kondrashuk and A. V. Gladyshev, Nucl. Phys. B471, 389 (1996), hep-ph/9511419.

[101] K. Yoshioka, Phys. Rev. D61, 055008 (2000), hep-ph/9705449].

[102] S. Hamidi and J. H. Schwarz, Phys. Lett. B147, 301 (1984).

[103] K. S. Babu, T. Enkhbat and I. Gogoladze, Phys. Lett. B555, 238 (2003), hep-ph/0204246.

[104] S. Heinemeyer, M. Mondragon and G. Zoupanos, JHEP 07, 135 (2008), [0712.3630].

[105] S. Heinemeyer, M. Mondragon and G. Zoupanos, Phys. Lett. B 718 (2013) 1430 arXiv:1211.3765 [hep-ph]].

[106] S. Heinemeyer, M. Mondragon and G. Zoupanos, Phys. Part. Nucl. 44 (2013) 299.

[107] M. S. Carena, D. Garcia, U. Nierste and C. E. M. Wagner, Nucl. Phys. B577, 88 (2000), hep-ph/9912516].

[108] K. Nakamura et al. [Particle Data Group], J. Phys. G 37 (2010) 075021. Update!

[109] Tevatron Electroweak Working Group for the CDF and D0 Collaborations, arXiv:1107.5255 [hep-ex].

[110] T. Kobayashi, J. Kubo, M. Mondragon and G. Zoupanos, Surveys High Energ. Phys. 16, 87 (2001).

[111] Heavy Flavour Averaging Group, see: http://www.slac.stanford.edu/xorg/hfag/.

[112] R. Aaij et al. [LHCb Collaboration], Phys. Rev. Lett. 110 (2013) 021801 arXiv:1211.2674 [Unknown]].

[113] H. Goldberg, Phys. Rev. Lett. 50 (1983) 1419; J. Ellis, J. Hagelin, D. Nanopoulos, K. Olive and M. Srednicki, Nucl. Phys. B 238 (1984) 453.

[114] E. Komatsu et al. [WMAP Collaboration], Astrophys. J. Suppl. 192 (2011) 18 arXiv:1001.4538 [astro-ph.CO]]; http://lambda.gsfc.nasa.gov/product/map/current/parameters.cfm. 
[115] M. Diaz, J. Romao and J. Valle, Nucl. Phys. B 524 (1998) 23 [arXiv:hep-ph/9706315]; J. Valle, arXiv:hep-ph/9907222 and references therein;

M. Diaz, M. Hirsch, W. Porod, J. Romao and J. Valle, Phys. Rev. D 68 (2003) 013009 [Erratum-ibid. D 71 (2005) 059904] arXiv:hep-ph/0302021].

[116] H. Dreiner, arXiv:hep-ph/9707435,

G. Bhattacharyya, arXiv:hep-ph/9709395;

B. Allanach, A. Dedes and H. Dreiner, Phys. Rev. D 60 (1999) 075014 arXiv:hep-ph/9906209;

J. Romao and J. Valle, Nucl. Phys. B 381 (1992) 87;

[117] D. Lyth and E. Stewart, Phys. Rev. D 53 (1996) 1784 arXiv:hep-ph/9510204.

[118] G. Gelmini and P. Gondolo, Phys. Rev. D 74 (2006) 023510 arXiv:hep-ph/0602230.

[119] G. Belanger, F. Boudjema, A. Pukhov and A. Semenov, Comput. Phys. Commun. 149 (2002) 103 arXiv:hep-ph/0112278; ;arXiv:hep-ph/0405253.

[120] S. Heinemeyer, W. Hollik and G. Weiglein, Comput. Phys. Commun. 124, 76 (2000), hep-ph/9812320].

[121] S. Heinemeyer, W. Hollik and G. Weiglein, Eur. Phys. J. C9, 343 (1999), hep-ph/9812472.

[122] G. Degrassi, S. Heinemeyer, W. Hollik, P. Slavich and G. Weiglein, Eur. Phys. J. C28, 133 (2003), hep-ph/0212020.

[123] M. Frank et al., JHEP 02, 047 (2007), hep-ph/0611326.

[124] T. Hahn, S. Heinemeyer, W. Hollik, H. Rzehak and G. Weiglein, Comput. Phys. Commun. 180 (2009) 1426.

[125] S. Heinemeyer, O. Stål and G. Weiglein, Phys. Lett. B 710 (2012) 201 arXiv:1112.3026 [hep-ph]].

[126] L. Hall, D. Pinner and J. Ruderman, JHEP 1204 (2012) 131 arXiv:1112.2703 [hep$\mathrm{ph}]]$;

H. Baer, V. Barger and A. Mustafayev, Phys. Rev. D 85 (2012) 075010 arXiv:1112.3017 [hep-ph]];

A. Arbey, M. Battaglia, A. Djouadi, F. Mahmoudi and J. Quevillon, Phys. Lett. B 708 (2012) 162 [arXiv:1112.3028 [hep-ph]];

P. Draper, P. Meade, M. Reece and D. Shih, Phys. Rev. D 85 (2012) 095007 arXiv:1112.3068 [hep-ph]].

[127] M. Carena, S. Gori, N. Shah and C. E. M. Wagner, JHEP 1203 (2012) 014 arXiv:1112.3336 [hep-ph]];

M. Carena, S. Gori, N. Shah, C. E. M. Wagner and L. -T. Wang, JHEP 1207 (2012) 175 [arXiv:1205.5842 [hep-ph]]; 
M. Carena, I. Low and C. E. M. Wagner, JHEP 1208 (2012) 060 arXiv:1206.1082 [hep-ph]];

M. Carena, S. Gori, I. Low, N. Shah and C. E. M. Wagner, arXiv:1211.6136 [hep-ph], to appear in JHEP.

[128] R. Benbrik, M. Gomez Bock, S. Heinemeyer, O. Stål, G. Weiglein and L. Zeune, Eur. Phys. J. C 72 (2012) 2171 arXiv:1207.1096 [hep-ph]];

P. Bechtle, S. Heinemeyer, O. Stål, T. Stefaniak, G. Weiglein and L. Zeune, arXiv:1211.1955 [hep-ph].

[129] M. Carena, S. Heinemeyer, O. Stl, C. E. M. Wagner and G. Weiglein, arXiv:1302.7033 [hep-ph].

[130] CMS Collaboration, S. Chatrchyan et al., Phys.Lett. B713, 68 (2012), [1202.4083].

[131] ATLAS Collaboration, P. Pravalorio, Talk at SUSY2012 (2012).

[132] CMS Collaboration, C. Campagnari, Talk at SUSY2012 (2012).

[133] M. Davier, A. Hoecker, B. Malaescu and Z. Zhang, 1010.4180.

[134] T. Moroi, Phys.Rev. D53, 6565 (1996), hep-ph/9512396.

[135] Muon G-2 Collaboration, G. Bennett et al., Phys.Rev. D73, 072003 (2006), hep-ex/0602035.

[136] P. Dirac, Lectures On Quantum Field Theory (1964).

[137] F. J. Dyson, Phys. Rev. 85, 631 (1952).

[138] S. Weinberg, 0903.0568, and references therein.

[139] S. Heinemeyer, M. Mondragon and G. Zoupanos, SIGMA 6, 049 (2010), [1001.0428]. 